\title{
Integrating cross-frequency and within band functional networks in resting-state MEG: A multi-layer network approach
}

\author{
a Sir Peter Mansfield Imaging Centre, School of Physics and Astronomy, University of Nottingham, Nottingham, United Kingdom \\ ${ }^{b}$ Department of Clinical Neurophysiology, MEG Center, VU University Medical Centre, Amsterdam, The Netherlands \\ ${ }^{c}$ Delft University of Technology, Faculty of Electrical Engineering, Mathematics and Computer Science, Delft, The Netherlands \\ d Oxford Centre for Human Brain Activity (OHBA), University of Oxford, Oxford, United Kingdom \\ e Centre for the Functional Magnetic Resonance Imaging of the Brain (FMRIB), University of Oxford, Oxford, United Kingdom
}

Prejaas Tewarie ${ }^{\mathrm{a}, *}$, Arjan Hillebrand ${ }^{\mathrm{b}}$, Bob W. van Dijk ${ }^{\mathrm{b}}$, Cornelis J. Stam ${ }^{\mathrm{b}}$, George C. O'Neill ${ }^{\mathrm{a}}$, Piet Van Mieghem ${ }^{\text {c }}$, Jil M. Meier ${ }^{c}$, Mark W. Woolrich ${ }^{\text {d,e }}$, Peter G. Morris ${ }^{\text {a }}$, Matthew J. Brookes ${ }^{\text {a }}$

\section{A R T I C L E I N F O}

\section{Article history:}

Received 29 February 2016

Accepted 27 July 2016

Available online 3 August 2016

\section{Keywords:}

Multi-layer networks

Interconnected functional networks

Functional connectivity

Cross-frequency coupling

Magnetoencephalography MEG

\begin{abstract}
A B S T R A C T
Neuronal oscillations exist across a broad frequency spectrum, and are thought to provide a mechanism of interaction between spatially separated brain regions. Since ongoing mental activity necessitates the simultaneous formation of multiple networks, it seems likely that the brain employs interactions within multiple frequency bands, as well as cross-frequency coupling, to support such networks. Here, we propose a multi-layer network framework that elucidates this pan-spectral picture of network interactions. Our network consists of multiple layers (frequency-band specific networks) that influence each other via inter-layer (cross-frequency) coupling. Applying this model to MEG resting-state data and using envelope correlations as connectivity metric, we demonstrate strong dependency between within layer structure and inter-layer coupling, indicating that networks obtained in different frequency bands do not act as independent entities. More specifically, our results suggest that frequency band specific networks are characterised by a common structure seen across all layers, superimposed by layer specific connectivity, and inter-layer coupling is most strongly associated with this common mode. Finally, using a biophysical model, we demonstrate that there are two regimes of multi-layer network behaviour; one in which different layers are independent and a second in which they operate highly dependent. Results suggest that the healthy human brain operates at the transition point between these regimes, allowing for integration and segregation between layers. Overall, our observations show that a complete picture of global brain network connectivity requires integration of connectivity patterns across the full frequency spectrum.
\end{abstract}

(c) 2016 Published by Elsevier Inc.

\section{Introduction}

Electrophysiological measurements of human brain activity are dominated by neuronal oscillations, observed across a wide range of temporal scales. This temporal richness allows analysis of oscillations in different frequency bands (e.g. alpha $(8-13 \mathrm{~Hz})$, beta $(13-30 \mathrm{~Hz})$ ) and there is good evidence that separate bands are responsible for different computational roles (da Silva, 2013). Oscillations are thought to play a core role in mediating synchronization between functionally specific brain regions, which is required to support cognitive processes (Engel et al., 2013; Hall et al., 2014; Schölvinck et al., 2013). Many studies have assessed inter-regional connectivity using electrophysiological

\footnotetext{
* Corresponding author at: Sir Peter Mansfield Imaging Centre, School of Physics and Astronomy, University of Nottingham, Nottingham, United Kingdom.

E-mail address: prejaas.tewarie@nottingham.ac.uk (P. Tewarie).
}

measurements, and the importance of such investigations is growing with good evidence of abnormal electrophysiological connectivity in disease (see e.g. (Stam, 2014; Tewarie et al., 2015)). To date, most studies probe network formation either within specific frequency bands (Brookes et al., 2011; Hipp et al., 2012) (e.g. alpha to alpha) or via specific cross-frequency interactions (e.g. alpha to beta) (Jensen and Colgin, 2007; Jiang et al., 2015; Watrous et al., 2015). The idea of network segregation across bands is supported by previous work, which has revealed, for example, formation of frequency specific task positive (O'Neill et al., 2015; O'Neill et al., 2016) and resting-state (Baker et al., 2014) networks. Furthermore, sensory processing seems to be supported by simultaneous formation of multiple networks mediated by separate bands. However, there is also evidence that efficient brain function not only relies on the formation of independent networks, but also on their integration (Deco et al., 2015). If neuronal oscillations provide a mechanism supporting this segregation and integration, it follows that global function must rely on a regime that facilitates 
segregation and integration of frequency specific networks. E.g. in a simplistic case of a beta band motor network and an alpha band visual network, a beta-to-alpha interaction might facilitate visuo-motor integration. Here, we introduce a generalized framework to assess oscillatory interactions; we will probe the relation between within and cross-frequency networks, and test a hypothesis that the brain maximizes efficiency by allowing formation of independent and integrative networks.

Insight into how brain network organisation might be mediated by within and between frequency oscillatory interactions can be obtained with the help of recent advances in network theory; specifically the field of interconnected or multi-layer networks (Boccaletti et al., 2014; Menichetti et al., 2014; Radicchi and Arenas, 2013). In the special case of multi-layer networks that we consider, every layer shares the same set of nodes (brain regions), but is characterised by its own set of links (intra-layer coupling) (Boccaletti et al., 2014). In addition, layers are coupled to each other by interactions known as inter-layer coupling (see Fig. 1). One of the aims of this field is to study the mutual interactions between networks: for example, one study modelled how an epidemic spreads across a network of people, (Granell et al., 2013), concluding that adding the awareness of the epidemic in the corresponding social network to the model allowed for a better description of realworld epidemic spread. Several other studies have also concluded that a multi-layer network model contains non-trivial information that cannot be retrieved by considering individual layers in isolation (Battiston et al., 2014; De Domenico et al., 2013; Martín-Hernández et al., 2014; Nicosia et al., 2013; Nicosia and Latora, 2014; Sahneh et al., 2015; Wang et al., 2013). In the present case of neuronal networks, we will use a framework in which separate layers represent within frequency band interactions, whereas cross-frequency interactions will be represented by inter-layer coupling (Fig. 1). In this way we analyse the network organisation across the electrophysiological spectrum in a single framework and use it to test the relationship between networks formed on the basis of within- and cross-frequency coupling. More specifically, recent theoretical network studies have revealed that for regular oneto-one or all-to-all inter-layer coupling, there exists an abrupt transition between two regimes of multi-layer network behaviour when interlayer coupling is systematically varied (Sahneh et al., 2015; Shakeri et al., 2015; Van Mieghem, 2016). In one regime, the individual layers are decoupled and operate independently, whereas in the second regime the individual layers are almost identical and the multi-layer network behaves as a single entity. We hypothesise that optimal brain network organisation should operate at the transition between these two extremes. This property of the multi-layer network would allow the different frequency specific networks to act independently, but also coherently, depending on the cognitive demand of the moment. Put another way, the existence of the global brain network at this transition would allow for segregation as well as integration of frequency specific networks.

A common way to analyse the potential transitional behaviour of neuronal networks is to link empirical neuroimaging data to largescale models of neuronal activity (Coombes, 2010; Deco et al., 2015). Most studies employ measured structural coupling (e.g. extracted from Diffusion Tensor Imaging) between spatially separate brain regions to define an underlying structural network. Activity within these separate regions is then simulated according to some oscillator model which mimics neuronal behaviour, and global coupling of the structural network is tuned until the modelled functional connectivity exhibits the best match to empirical data. Previous (magnetoencephalography) MEG studies (Cabral et al., 2014; Nakagawa et al., 2014; Tewarie et al., 2014) have shown that within band connectivity patterns in empirical MEG data hint at a weak structural coupling near an abrupt transition for synchronization. In the current study we will combine such a model with source localised (MEG) data in order to test our prediction of an operating point for MEG multi-layer networks near a transition with respect to structural coupling strength. In what follows, we first use empirical (resting-state) MEG data in 31 healthy subjects to better understand the relationship between within layer and between layer couplings. Specifically, we demonstrate a significant relationship between layer similarity and inter-layer coupling. Secondly, we show that separate layers are characterised by a common spatial structure, which is overlaid by frequency specific networks with distinct spatial multi-layer network

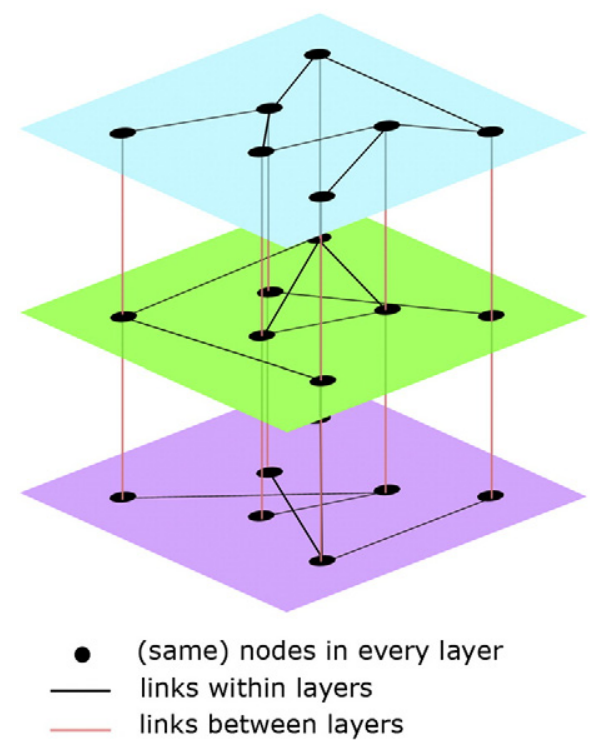

\section{MEG multi-layer network}

Layer 1

Layer 2

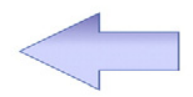

Layer 3

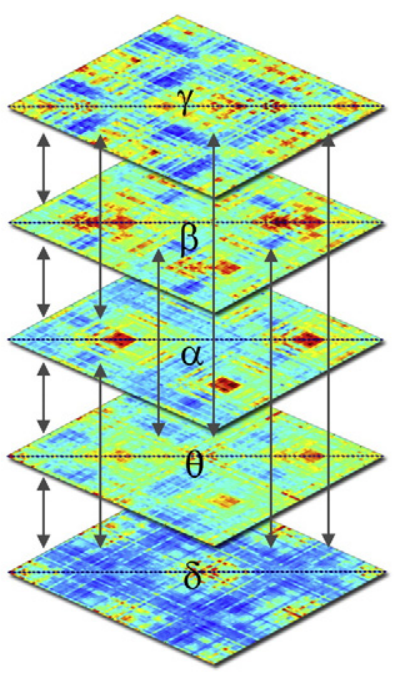

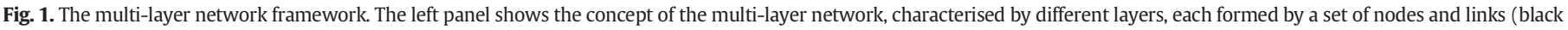

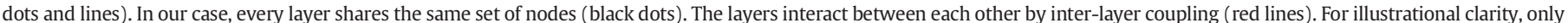

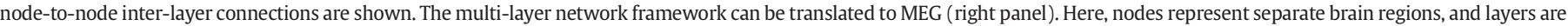

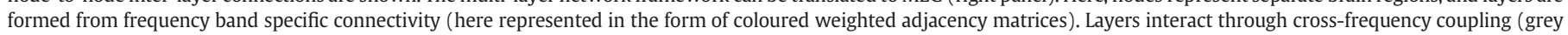
arrows). 
topographies. Finally, we combine empirical data with a whole brain model (Robinson et al., 2001) to test our primary hypothesis that the brain, when modelled as a multi-layer network, operates in the vicinity of a transition that separates two regimes of behaviour, facilitating integration and segregation of networks mediated by dependency between frequency bands.

\section{Theory}

We consider a special case of a multi-layer or interconnected network, where each layer shares the same set of nodes (brain regions), but where the link weight structure (connection strengths) within each layer can be different. We will consider weighted and fully connected networks within each layer, i.e. complete graphs. A single layer, $f$, (with $f \in\{1,2, \ldots, M\}$ ) represents a frequency band specific network. Here we consider five standard frequency bands commonly used in resting-state MEG analysis ( $f=1$ : delta $1-4 \mathrm{~Hz}, f=2$ : theta 4-8 Hz, $f=3$ : alpha $8-13 \mathrm{~Hz}, f=4$ : beta $13-30 \mathrm{~Hz}$ and $f=5$ : gamma $30-48 \mathrm{~Hz}$ ); i.e. $M=5$ layers. Each layer consists of $N(N=78$ (Gong et al., 2009)) nodes and $E$ links (where $E=1 / 2\left(N^{2}-N\right)$ ) and can be described by a weighted symmetric adjacency matrix $W_{f}(N x N$ matrix) with zeros on the diagonal. These layers can be combined to form the multi-layer network, which is characterised by a weighted blockadjacency matrix, which comprises the $M$ intra-layer networks as blocks along its diagonal as well as the (generally non-symmetric) inter-layer coupling matrices $H_{l m}(N \times N$ matrix), where $l$ and $m$ are indices of the layers, which form the off-diagonal blocks. For example, the interlayer coupling matrix $\mathrm{H}_{23}$ on the off-diagonal means the coupling between the second (theta) and third (alpha) layer and thus represents theta to alpha coupling between all possible node pairs. Mathematically, the block-adjacency matrix is given as (Sahneh et al., 2015)

$\boldsymbol{W}=\operatorname{diag}\left(W_{f}\right)+(H \otimes B)$

with

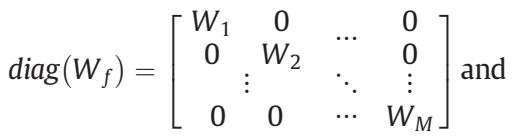

$$
\begin{aligned}
& H=\left[\begin{array}{cccc}
0 & H_{12} & \ldots & H_{1 M} \\
H_{21} & 0 & \cdots & H_{2 M} \\
& \vdots & \ddots & \vdots \\
H_{M 1} & H_{M 2} & \cdots & 0
\end{array}\right]
\end{aligned}
$$

where $\otimes$ is the matrix Kronecker product (Van Mieghem, 2010). The first term on the right-hand side of Eq. (1a) $\left(\operatorname{diag}\left(W_{f}\right)\right)$ is a diagonal block matrix with the matrices $W_{f}$ on the diagonal and matrices consisting of zeros (with equal size) on the off-diagonals. The second term $(H \otimes B)$ reflects the cross-frequency interactions which, in MEG, can be derived from e.g. phase-phase, phase-amplitude or amplitudeamplitude interactions. The matrix $B(N \times N$ matrix $)$ designates the inter-layer connections of interest. For example, $B=I$ would indicate that the inter-layer connection patterns are one-to-one: meaning that node $i$ in layer $l$ is only connected to node $i$ in layer $m$ (see Fig. 1). In our case we have placed no limit on this masking matrix $B$, (i.e. $B$ is a matrix of all-ones) meaning that any node in one layer can exert influence over any node (including itself) in any other layer. Thus in our case, $\boldsymbol{W}$ consists of 5 frequency specific weighted adjacency matrices on the diagonal and cross-frequency weighted adjacency matrices on the off-diagonals. It is instructive at this point to outline a set of useful metrics that can be calculated directly from the block-adjacency matrix; these important metrics will be used throughout this paper:
- $\langle P\rangle$ represents the overall strength of coupling between all layers in the multi-layer network. It is given by the mean of all the elements of the off-diagonal blocks (i.e. mean of all elements in the second term in Eq. (1a)) thus:

$$
\langle P\rangle=\frac{1}{\left(M^{2}-M\right)} \sum_{l=1}^{M} \sum_{m=1, m \neq l}^{M}\left\langle H_{l m} B\right\rangle,
$$

where $\langle X\rangle=u^{T} X u / N^{2}$ indicates the mean of the $N \times N$ matrix $X$ over all elements, where $u$ is the all-one vector, $\mathrm{u}=[1,1, \ldots, 1]^{\mathrm{T}}$.

- $\langle W\rangle$ represents the overall strength of the coupling within layers in the multi-layer network model. It is given by the mean of the main diagonal blocks of the block-adjacency matrix

$$
\langle W\rangle=\frac{1}{M} \sum_{f=1}^{M}\left\langle W_{f}\right\rangle
$$

- $C(X, Y)$ represents the block correlation which is given simply by the Pearson correlation coefficient measured between the upper triangular part of the weighted adjacency matrices $X$ and $Y$; e.g. $C\left(W_{2}, W_{5}\right)$ quantifies the similarity between connectivity measured in the theta band and connectivity measured in the gamma band. The correlations are computed at the link level, and not at the node level.

- Lastly, we are interested in the eigenvalues (spectrum) of the blockLaplacian matrix (Van Mieghem, 2010). The general form for the Laplacian matrix is $\left(L_{f}=D_{f}-W_{f}\right)$, where $D_{f}$ is a diagonal matrix with the node-strengths or degrees of $W_{f}$ on the diagonal (Sahneh et al., 2015). Such a Laplacian matrix can also be derived for the multilayer network as a whole. This block-Laplacian matrix can be obtained (Sahneh et al., 2015) from Eq. (1a) and is written as

$\boldsymbol{L}=\operatorname{diag}\left(L_{f}\right)+\left(L_{H} \otimes B\right)$,

where

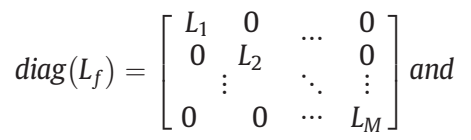

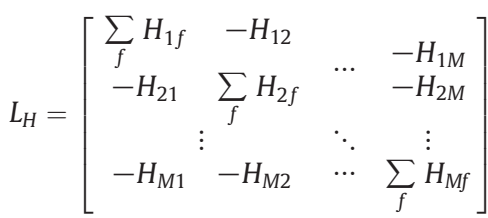

Here $L_{f}$ (with $f \in\{1,2, \ldots, M\}$ ) are the Laplacian matrices for the various layers and $B$ has the same meaning as above. The diagonal elements in $L_{H}$ can be computed as indicated in Eq. (4b) since the row and column sum of any Laplacian is zero, and the diagonal elements are equal to the absolute value of the sum over the columns (Van Mieghem, 2010). The rationale to investigate the Laplacian spectrum is the following: previous studies have shown that by analysing the behaviour of the eigenvalues of the block-Laplacian matrix, specifically the second smallest eigenvalue (algebraic connectivity $\lambda_{2}$ ), two regimes of multi-layer network behaviour can be identified, one in which network layers act independently, and the second in which network layers are coupled strongly (Gomez et al., 2013; Martín-Hernández et al., 2014; Sahneh et al., 2015; Shakeri et al., 2015). Importantly, a recent theoretical study has derived analytical expressions for this sharp transition behaviour between the two regimes (Van Mieghem, 2016), which can be observed when the inter-layer coupling is regular, i.e. for networks with an inter-layer coupling structure of $B=w J$ (with $w=$ 1) and with $\langle P\rangle=1$. Given the absence of analytical expressions for our case (where $\langle P\rangle \ll 1$ (see Fig. 2D)), we have to follow an 
exploratory numerical approach by analysing the spectrum (and specifically $\lambda_{2}$ ) of empirical MEG multi-layer networks in relation to the simulated data based on whole brain network models (see Sections Network simulations based on a cortico-thalamic mean-field model and Agreement between simulations and empirical data). Note that, similar to single layer networks, the eigenvalues of the Laplacian matrix of multi-layer networks, including $\lambda_{2}$, correspond to several dynamic and topological properties of the network as a whole, ranging from synchronization properties (Sole-Ribalta et al., 2013), epidemic thresholds (Wang et al., 2013) to robustness of the multilayer network as a whole (Sánchez-García et al., 2014; Van Mieghem, 2016). However, the interpretation of specific eigenvalues of multilayer networks is not for every case straightforward and needs further theoretical investigation.

\section{Methods}

Empirical MEG data acquisition, pre-processing and source localisation

The data collection and pre-processing steps of the current dataset have been described in detail elsewhere (Tewarie et al., 2016) and here we include a summary for completeness. Thirty-one healthy control subjects (age $27.4 \pm 6.4$ (mean and standard deviation), 40\% female) with no history of neurological impairment were enrolled and scanned as part of the University of Nottingham's Multi-modal Imaging Study in Psychosis. The study was approved by the University of Nottingham Medical School Ethics Committee, and all subjects gave written informed consent prior to participation. MEG data were acquired using the third order synthetic gradiometer configuration of a 275 channel CTF MEG system (CTFMEG, Canada), at a sampling rate of $600 \mathrm{~Hz}$ and using a $150 \mathrm{~Hz}$ low pass anti-aliasing filter. Magnetic fields were recorded during a taskfree, eyes-open condition for $10 \mathrm{~min}$ with all volunteers in a supine position. Subjects were asked to fixate on a red cross throughout the experiment, which was displayed via back projection on a screen placed $\sim 46 \mathrm{~cm}$ in front of the subject. Three coils were attached to the participant's head as fiducial markers at the nasion, left and right preauricular points. These coils were energised periodically throughout acquisition to allow localisation of the head relative to the geometry of the MEG sensor array. Before MEG acquisition, the surface of the participant's head was digitised using a 3D digitiser (Polhemus Inc.). Subsequent surface matching of the digitised head shape to an equivalent head shape extracted from an anatomical magnetic resonance (MR) image (see below for acquisition details) allowed coregistration of brain anatomy to MEG sensor geometry. Following collection, MEG data were inspected for artefacts generated by, for example, the magnetomyogram, magnetooculogram and magnetocardiogram. Any trials deemed to
A

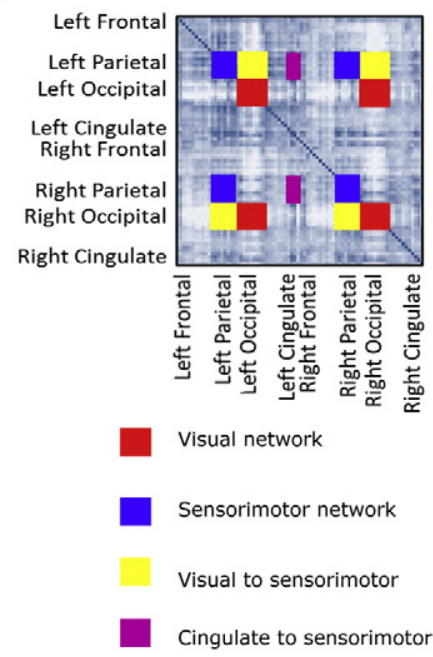

B
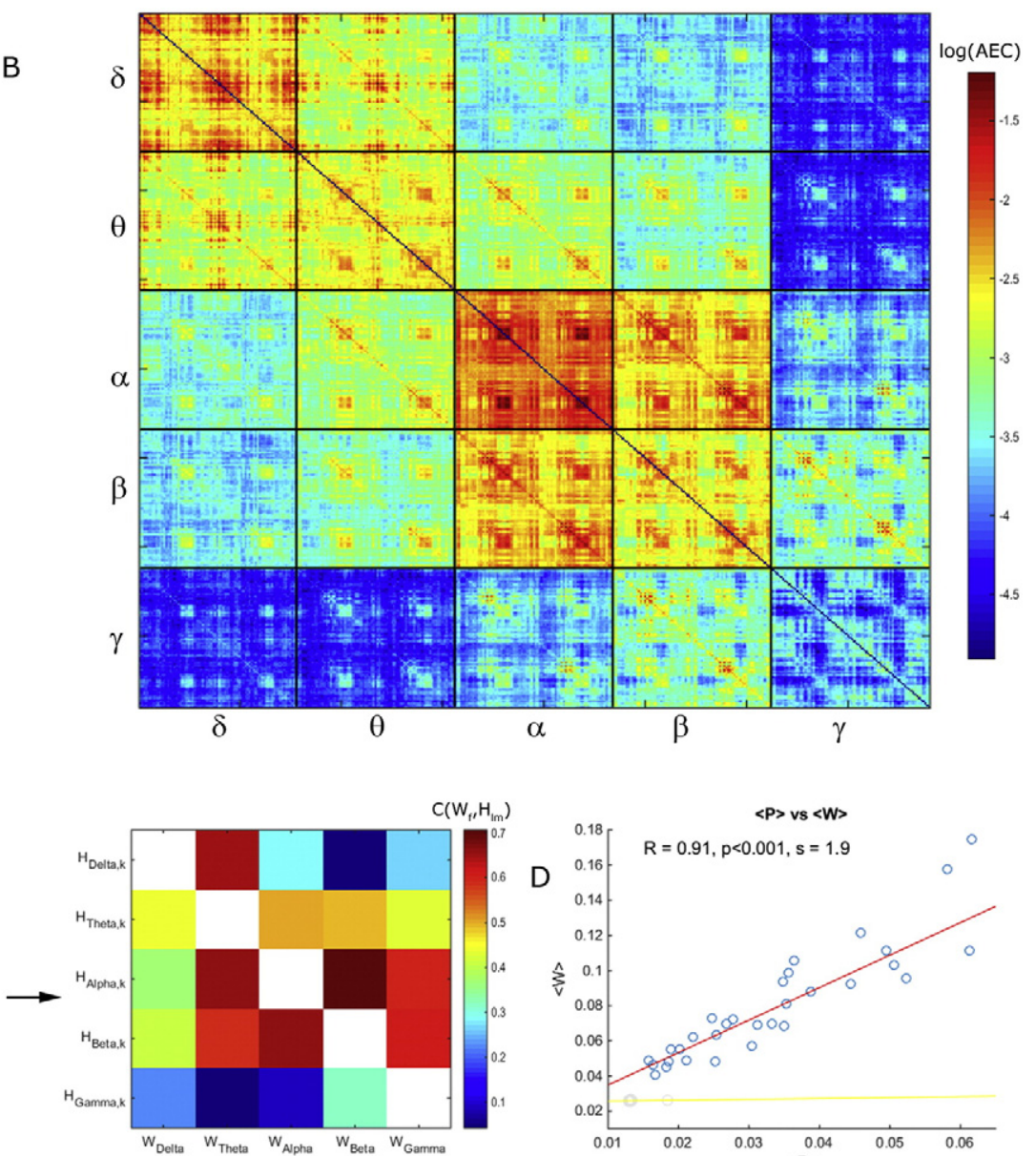

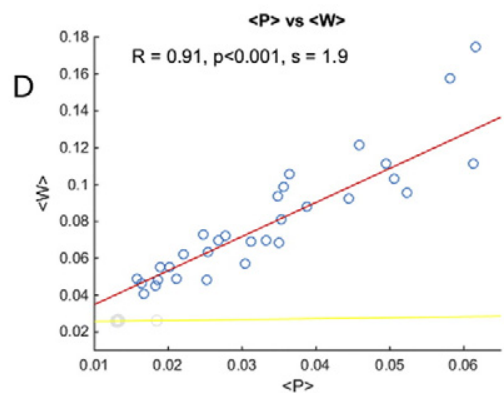

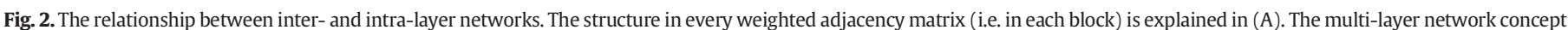

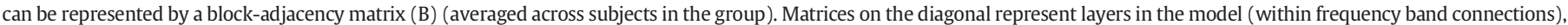

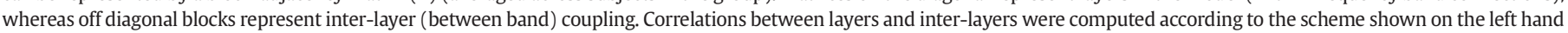

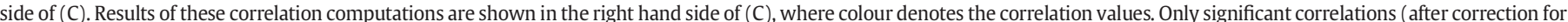

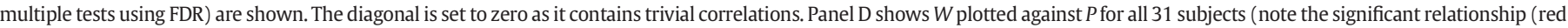

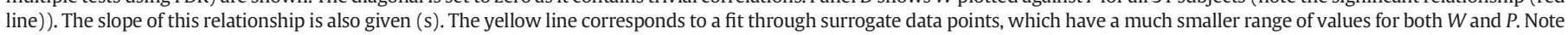
that those individuals with high within layer connectivity $W$ also tend to have high between layer connectivity $P$. 
contain excessive interference were removed. In addition, trials in which the participant was found to have moved $>7 \mathrm{~mm}$ from their starting position were also removed.

An atlas-based beamforming approach (Hillebrand et al., 2012), equivalent to that used in (Tewarie et al., 2016) was used for source space localisation. Briefly, the cortex was parcellated using the automated anatomical labelling (AAL) atlas (Tzourio-Mazoyer et al., 2002). From the 116 AAL regions, we only selected 78 cortical regions covering the entire cortex and ignored the 38 remaining subcortical and cerebellar regions. A beamformer approach (Robinson and Vrba, 1999) was employed to generate a single timecourse of electrophysiological activity within each of these regions. To achieve this, for each region, first the centre of mass was derived. Given the spatial resolution of MEG (a few $\mathrm{mm}$; (Barnes et al., 2004)), voxels were defined on a regular $4 \mathrm{~mm}$ grid covering the entire region, and the beamformer estimated timecourse of electrical activity was derived for each voxel. To generate a single timecourse representing the whole region, individual voxel signals were weighted according to their distance from the centre of mass using a Gaussian weighting function. This ensures that the regional timecourse is biased towards the centre of the region, with a full width at half maximum of $\sim 17 \mathrm{~mm}$. To calculate individual voxel timecourses, a scalar beamformer was used (Robinson and Vrba, 1999). Covariance was computed within a $1-150 \mathrm{~Hz}$ frequency window and a time window spanning the whole experiment (Brookes et al., 2008). Regularisation was applied to the data covariance matrix using the Tikhonov method with a regularisation parameter equal to $5 \%$ of the maximum eigenvalue of the unregularised covariance matrix. The forward model was based upon a dipole approximation (Sarvas, 1987) and a multiple local sphere head model (Huang et al., 1999). Dipole orientation was determined using a non-linear search for optimum signalto-noise ratio (SNR). Beamformer timecourses were sign-flipped where necessary in order to account for the arbitrary polarity introduced by the beamformer source orientation estimation. This complete process resulted in 78 electrophysiological timecourses, each representative of a separate AAL region. This approach was applied to each subject individually.

\section{Construction of multi-layer MEG networks}

Functional connectivity was estimated as correlation between the envelopes of band limited oscillations (often termed amplitude envelope correlation - AEC). This method has been used extensively in previous studies to characterize long-range interactions between brain regions (Hipp et al., 2012; O'Neill et al., 2015). The AEC was computed between all possible pairs of beamformer projected regional timeseries for each subject separately. Beamformer projected data were filtered into five frequency bands: delta $(1-4 \mathrm{~Hz})$, theta $(4-8 \mathrm{~Hz})$, alpha $(8-13 \mathrm{~Hz})$, beta $(13-30 \mathrm{~Hz})$, gamma $(30-48 \mathrm{~Hz})$. The following steps were then taken to compute AEC:

1) Pairwise orthogonalisation of time-series by means of linear regression in order to reduce the effects of signal leakage (e.g. (Brookes et al., 2012; Hipp et al., 2012)).

2) Computation of the envelope by calculating the absolute value of the analytical signal, obtained by a Hilbert transformation.

3) Temporal down-sampling (to $1 \mathrm{~s}$ ) applied to the envelopes as described in a previous paper (Brookes et al., 2011).

4) Pearson correlation computed between temporally down-sampled envelope pairs, with each correlation coefficient forming a single element in the weighted adjacency matrix.

These steps were performed for all possible pairs of time-series within a frequency band to obtain intra-layer weighted adjacency matrices, $W_{f}$. Each $W_{f}$ was then transformed by taking the absolute value of all elements in order to avoid cancellation within and across layers when computing $W$. Similar steps were followed to assess between frequency band interactions; these resulted in the off-diagonal weighted adjacency matrices (inter-layer networks) $H_{l m}$. The orthogonalisation procedure for between frequency connectivity was maintained in order to correct for any spectral leakage. Again the absolute value of all elements in $H_{l m}$ was computed to allow unambiguous computation of $P$. The block-adjacency matrix $\boldsymbol{W}$ and the block-Laplacian matrix $\boldsymbol{L}$ were derived by inserting these inter-layer and intra-layer networks into Eqs. (1) and (4), respectively.

\section{Network simulations based on a cortico-thalamic mean-field model}

Similar to previous studies (e.g. (Deco et al., 2013b; Freyer et al., 2011)) we employed a cortico-thalamic mean-field model. The advantage of using this cortico-thalamic mean-field model is that, unlike in empirical data, there exists a ground truth which allows us to address the question of whether MEG networks may operate in the vicinity of a transition point that separates two regimes of multi-layer network behaviour. We used a cortico-thalamic mean-field model that describes the mean dynamics of large populations of neurons (Robinson et al., 2002; Robinson et al., 2004; Robinson et al., 2001). This model is optimised to produce realistic power spectra mimicking real EEG/MEG data. Here, we considered a network of cortico-thalamic units where a unit was defined by a cortical and a thalamic population, with the cortical population formed by an inhibitory and excitatory neuronal group and the thalamic population by a thalamic reticular and a thalamocortical relay group. We denote these groups as $a \in\{e, i, r, s\}$, where $e, i, r, s$ denote excitatory, inhibitory, reticular and relay respectively. For each group, $a$, and region $j$ the mean membrane potential is denoted by $V_{a, j}$ and the mean firing rate by $Q_{a, j}$, which were interrelated by a sigmoid function:

$$
Q_{a, j}=\frac{Q_{\max }}{\left.1+\exp \left(-\left(V_{a, j}-\theta\right) / \sigma\right)\right)}
$$

Here, $Q_{\max }$ refers to the maximum firing rate in $\mathrm{Hz}, \theta$ is the mean firing threshold in $m V$, and $\sigma$ is the standard deviation of this threshold. The mean membrane potential $V_{a, j}$ itself fluctuates under the influence of incoming firing input from other groups within the same population $\left(Q_{a, j}, a \in\{e, i, r, s\}\right)$ as well as from other excitatory regions belonging to other populations, $\phi_{e, n}$, outside its own region. The mean membrane potential $V_{a, j}$ can be considered as a low-pass filter. For every group its dynamics can be described by

$$
\begin{aligned}
& D_{a} V_{e, j}(t)=v_{e e} \phi_{e, j}(t)+v_{e i} Q_{i, j}(t)+v_{e s} Q_{s, j}\left(t-\tau_{c t}\right) \\
& +\epsilon \frac{1}{N} \sum_{n=1, n \neq j}^{N} A_{j n} \phi_{e, k}\left(t-\tau_{j n}\right) \\
& D_{a} V_{i, j}(t)=v_{i e} \phi_{e, j}(t)+v_{i i} Q_{i, j}(t)+v_{e s} Q_{s, j}\left(t-\tau_{c t}\right) \\
& D_{a} V_{s, j}(t)=v_{s e} \phi_{e, j}\left(t-\tau_{c t}\right)+v_{s r} Q_{r, j}(t)+v_{n} \phi_{n, j}(t) \\
& D_{a} V_{r, j}(t)=v_{r e} \phi_{e, j}\left(t-\tau_{c t}\right)+v_{r s} Q_{s, j}(t) \\
& D_{a}=\frac{1}{\alpha \beta} \frac{d^{2}}{d t^{2}}+\left(\frac{1}{\alpha}+\frac{1}{\beta}\right) \frac{d}{d t}+1
\end{aligned}
$$

Here $\alpha$ and $\beta$ are constants, which are independent of time and correspond to the synaptic rise and decay rates in $s^{-1} ; v_{a a}$ denotes synaptic densities between the population types and $\epsilon$ corresponds to the global structural coupling strength between populations. The last term in Eq. (6c) corresponds to noise input, which is defined as $\phi_{n j}=\sigma_{n} \alpha \beta \chi(t)$, where $\chi(t)$ denotes a unit variance Gaussian white-noise process, and $\sigma_{n}$ the strength of this process. External firing input from other populations is mediated by the presence of an structural connection $A_{j n}$, where $A$ denotes a $78 \times 78$ adjacency matrix of a literature based structural network (Gong et al., 2009). Input from the thalamus to the cortex 
and vice versa is delayed by $\tau_{c t}$, and cortico-cortical excitatory input between regions is also mediated with a delay $\tau_{j n}$, depending on the Euclidian distance in the AAL atlas between region $j$ and $n$. As incoming excitatory firing input from other populations $Q_{e, n}$ is propagated over a long range, its initial activity $Q_{e, n}$ is damped by the following expression

$$
\left(\frac{1}{\gamma^{2}} \frac{d^{2}}{d t^{2}}+\frac{2}{\gamma} \frac{d}{d t}+1\right) \phi_{e, n}(t)=Q_{e, n}(t)
$$

The constant $\gamma$ refers to the cortical damping rate. Eq. (7) usually has an extra term on the left hand side, which contains the differential Laplace operator $\nabla^{2}$ (Robinson et al., 2001). Similar to Robinson et al. (2002) we ignore the spatial dynamics by setting $\nabla^{2}=0$ and therefore all observables in the model become independent of position. Note that there is still regional dependence based on the incoming connections over the white matter tracts in the network (Eq. (6a)). Values for all constants can be found in Table S1 and are the same parameters as used to simulate resting state activity in previous studies (Hindriks and van Putten, 2013; Robinson et al., 2002), with the only exception that we increased $v_{e i}$ to account for the extra excitatory input from the network. Simulations based on the cortico-thalamic mean-field model were performed using the Euler-Maruyama method with an integration time step of $1 \times 10^{-4}$. The first $2 \mathrm{~s}(20,000$ samples $)$ of the simulated data were discarded to exclude any non-oscillatory data, and the remaining simulated data were of equal duration (same number of samples) to the empirical data (600 s). The simulations were performed for a range of structural coupling strengths $\epsilon$. This procedure was executed 31 times (same as the number of subjects for the empirical data) and results averaged over realisations. The time series of the 78 excitatory populations $\phi_{e, n}(t)$ were used as the model output to mimic MEG signals (Robinson et al., 2002), i.e. only the activity from the excitatory population from the model is taken as representative time-series for the of the MEG signal. AEC was computed, in the same way as for empirical data (but without correction for signal leakage), to obtain simulated within frequency networks $W_{f}$ and between frequency band networks $H_{m l}$.

\section{Analysis steps}

Relationship between inter- and intra-layer networks

To test for an association between intra- (within frequency band) and inter- (between frequency band) layer networks in empirical MEG data, we undertook two separate tests. Firstly, we quantified the spatial relationship between separate blocks of the block-adjacency matrix as $C(X, Y)$ (see above). Specifically we tested for a significant relationship between the layers, $W_{f}$, and the inter-layer couplings, $H_{m l}$. The Pearson correlation coefficient between $W_{f}$ and $H_{f l}, C(X, Y)$ was computed at the group level, meaning that we averaged $W_{f}$ and $H_{f}$ across subjects prior to calculating spatial correlations. Secondly, we tested whether the mean strength of within frequency band connections, $W$, was correlated with the mean strength of cross-frequency connections, $P$. This was calculated as a relationship across subjects, meaning that $W$ and $P$ were estimated for each subject and then correlation across all 31 subjects was measured. Statistical analyses for both tests were carried out using an empirical null distribution (see Statistical testing section).

\section{Eigenvalue decomposition of the layers}

If our primary hypothesis of an operating point close to a transition between MEG multi-layer regimes is correct, it is reasoned that the network topographies observed in individual layers might be characterised by some common structure (i.e. a network common to all frequency bands), overlaid by frequency band specific interactions. Here, similarities between structure in the different layers were analysed using graph spectral theory, where each network-layer was decomposed and analysed at the level of its corresponding set of eigenvalues (spectrum) and eigenvectors (Van Mieghem, 2010). The information contained in the eigenvalues is linked to topological features of a network, ranging from stability or robustness of a network to synchronization properties (Dorogovtsev et al., 2003). The eigenvectors contain valuable information, e.g. on the community structure (Newman, 2006a, 2006b), topological structure (Wang and Van Mieghem, 2015) and also provide a set of centrality metrics for nodes (Van Mieghem, 2014). For a trivial interpretation in relation to the intra-layers we decomposed the symmetric weighted adjacency matrix, $W_{f}$

$W_{f}=V_{f} \Lambda_{f}\left(V_{f}\right)^{T}$,

where $V_{f}=\left[v_{f, 1} v_{f, 2} \ldots v_{f, N}\right]$ and contains the eigenvectors of $W_{f} . \Lambda_{f}$ is a diagonal matrix containing the eigenvalues $\lambda_{f, i}(i \in\{1,2, \ldots N\})$ of $W_{f}$. For each eigenvector and eigenvalue, we computed the outer product of an eigenvector with itself multiplied with its corresponding eigenvalue, which we call 'eigenmode product' defined as (Liu et al., 2010)

$Y_{f, i}=\lambda_{f, i} v_{f, i}\left(v_{f, i}\right)^{T}$ obeying $\sum_{i=1}^{N} Y_{f, i}=W_{f}$

Since all the eigenvectors (from a single symmetric weighted adjacency matrix) are orthogonal, the eigenmode products correspond to independent patterns derived from each eigenvector, weighted by its corresponding eigenvalue. If frequency band specific networks are characterised by common structure, they will share similar eigenmode products, whilst frequency band specific structure would be characterised by independence between eigenmode products (see below). In the case of an operating point close to a transition between multi-layer regimes, we would predict that some eigenmode products would be shared between layers, whereas some would be independent. We expect that the combination of shared and common modes would not be present in surrogate data (see below).

To test this quantitatively, we first computed Pearson correlations between separate layers, $C\left(W_{l}, W_{k}\right)$ as described previously. Secondly, we computed Pearson correlations between eigenmode products, $C\left(Y_{l i}, Y_{k i}\right)$. Finally, we quantified how much each eigenmode product contributes to the overall similarity between layers by computing the correlation between $W_{k}^{\prime}$ and $W_{l}^{\prime}$, denoted $C\left(W_{f}^{\prime}, W_{l}^{\prime}\right)$. Here $W_{k}^{\prime}$ and $W_{l}^{\prime}$ refer to weighted adjacency matrices with one eigenmode product removed:

$W_{l}^{\prime}=V_{f} Z_{f}\left(V_{f}\right)^{T}$ with $Z_{f}=\left[\lambda_{f, 1} \lambda_{f, 2} \ldots 0 \ldots \lambda_{f, N}\right]$

meaning that $Z_{f}$ is equivalent to $\Lambda_{f}$, but with $\lambda_{f, i}=0$ so as to remove the $i$ th eigenmode. Finally, we probed whether inter-layer coupling mediates similarity between layers, and how this is affected by the removal of specific eigenmode products. We computed Pearson correlations between the mean inter-layer coupling $P$ and similarity between layers, where layer similarity was calculated 1$)$ between the original layer matrices, $C\left(W_{l}, W_{f}\right) ; 2$ ) between eigenmode products, $C\left(Y_{l i}, Y_{f}\right)$; and 3 ) between residual matrices following eigenmode product removal, $C\left(W_{l^{\prime}}, W_{f}^{\prime}\right)$. In all 3 cases layer similarity was averaged across all layer pairs. Again all statistical analyses were completed using empirical null distributions (Statistical testing section).

\section{Agreement between simulations and empirical data}

To further test whether MEG multi-layer networks could be found near a transition between two regimes of behaviour, we analysed the relationship between empirical data and data simulated by the corticothalamic mean-field model described in (Network simulations based on a cortico-thalamic mean-field model section). Simulations were performed for a variety of different values of global structural coupling strength $\epsilon$, merely to adjust overall synchronisation-levels in the functional networks. For each $\epsilon$ we computed both $P_{\text {sim }}$ ( $\operatorname{sim}=$ simulations) 
and the spectrum of the block-Laplacian based on the simulations. We determined the coupling strength, $\epsilon$, at which $P_{\text {sim }}$ and the Laplacian spectrum obtained from simulations were most similar to the empirical values of $P$ and the Laplacian spectrum derived from the MEG data. Our hypothesis was that this would occur in a weakly coupled regime (Deco et al., 2013b) where we would expect transition behaviour between low and high $P$. We quantified the similarity between simulation and empirical MEG data in two ways. Firstly, we computed the Pearson correlation between the empirical and simulated block-adjacency matrices. Secondly, we compared the distributions of the empirical and simulated $P$ and spectra by computing the Kullback-Liebler divergence $\rho_{K L}$, which quantifies the dissimilarity between two distributions by.

$\rho_{K L}(g, f)=\int g(x) \log \left(\frac{g(x)}{f(x)}\right) d x$

Here $g(x)$ and $f(x)$ are the two distributions of interest; in our case $g(x)$ represents the distribution of $P$ and spectra across subjects and $f(x)$ represents the distribution of $P_{\text {sim }}$ and spectra across realisations of the simulation. To obtain a measure of similarity, we computed $1 / \rho_{K L}$ as a goodness-of-fit (GOF) estimate. Here, a high GOF corresponds to a high similarity between experimental and simulated data.

\section{Integration and segregation between layers}

To quantify integration and segregation of the separate layers, we computed a metric that has recently been described in Battiston et al. (2014). This metric (a conditional probability) quantifies the probability that links in layer $f$ are there given the presence of links in layer $l$ and is given by:

$P\left(W_{f, i j} \mid W_{l, i j}\right)=\frac{\sum_{i j} W_{f, i j} W_{l, i j}}{\sum_{i j} W_{l, i j}}$

If layers show integration, then $P\left(W_{f, i j} \mid W_{l, i j}\right)$ is high, whereas if layers are characterised by segregation, patterns in the layers become independent and $P\left(W_{f, i j} \mid W_{l, i j}\right)$ will be low.

\section{Statistical testing}

In all of the above analyses, statistical testing was undertaken via the generation of surrogate data, in order to test whether the empirical findings could be obtained in the absence of non-linear functional coupling. Surrogate MEG data were generated by a widely applied phase randomisation procedure (Allen et al., 2014; Brookes et al., 2014; Prichard and Theiler, 1994; Zalesky et al., 2012) to the frequency filtered beamformer derived (non-envelope) timecourses. This randomisation was done separately for each subject. In using this method it is important to note that both the Fourier spectrum of each regional timecourse, and the covariance structure of the original data is maintained. However, the phase relationships between timecourses are disrupted and this removes coherence between band-limited envelopes. In order to test statistically whether the obtained relationships/distributions in the analyses described in Relationship between inter- and intra-layer networks section and Integration and segregation between layers section were significant, we compared these relationships/distributions to null distributions obtained by repeating the analysis 500 times on surrogate data. Empirical values were deemed significant if these values were found in the upper tail of distributions ( $5 \%$ significance level). When multiple correlations were computed, we corrected for multiple tests with a false discovery rate (FDR) correction (Benjamini and Hochberg, 1995). In cases where we tested for an increase in empirical correlation, we repeated exactly the same correlations for the surrogate data and tested whether the change in correlation of the empirical data fell within the upper tail of the equivalent change in correlation for the surrogate data.

\section{Results}

\section{Relationship between inter- and intra-layer networks}

The full block-adjacency matrix, averaged across all 31 subjects, is shown in Fig. 2B. The diagonal blocks show the separate layers, $W_{f}$ and the off-diagonal blocks show inter-layer couplings $H_{l m}$. We have placed no limit on the masking matrix, $B$, (i.e. $B=J$ is a matrix of all-ones) meaning that any node in one layer can exert influence over any node (including itself) in any other layer. It is clear from Fig. 2B that within layer networks exhibit stronger interactions than inter-layer networks (with the exception of gamma), with the highest connectivity values observed in the alpha-to-alpha block. However, all blocks, including between layer couplings, show a clear structure in terms of the brain regions implicated. For example, the alpha-alpha layer shows high connectivity in the occipital cortex whilst beta-beta shows strong sensorimotor network interactions. Although cross-frequency interactions have been less closely studied in previous work, it is interesting to note that, for example, the beta to gamma inter-layer network shows strong (inter-hemispheric) connections between sensorimotor regions. The observation that beta to gamma interactions are stronger than within band gamma connections may be related to the higher signal-to-noise in the beta band compared to the gamma band.

Group-level correlations between layers $\left(W_{f}\right)$ and inter-layers $\left(H_{l m}\right)$ are illustrated in Fig. 2C. The right hand panel displays correlations between blocks, and only those correlations deemed significant with respect to the null-distributions are shown. The left hand panel in Fig. $2 \mathrm{C}$ shows the scheme for computing correlations; the arrows show which pair of matrices have been correlated, so for example the bottom left matrix element in the right hand panel corresponds to the relationship between $W_{\text {delta }}$ (within frequency coupling in the delta band) and $H_{\text {delta } \rightarrow \text { gamma }}$ (delta to gamma band interactions). All matrix pairs studied correlated significantly, with especially strong correlation between layers and inter-layers corresponding to neighbouring frequency bands (e.g. theta-alpha, alpha-beta, beta-gamma). This shows clearly that patterns of connectivity observed within specific bands are related to patterns of connectivity observed between frequencies. The relationship between within and between frequency coupling is further elucidated in Fig. 2D. Here $P$ (mean inter-layer coupling) and $W$ (mean intra layer coupling) are plotted for each individual subject. There is a strong correlation $(R=0.91$, comparison to null-distribution $p<0.001$ ) showing that subjects with high within layer coupling tend to also exhibit high inter-layer coupling. Taken together, results in Fig. 2B, C and D show that inter-layer (between frequency band) coupling exhibits significant spatial structure across brain regions. Furthermore, these between frequency band interactions are significantly correlated with the (more commonly measured) within layer (band specific) interactions in terms of both their spatial signature (Fig. 2C) and the magnitude of the observable coupling (Fig. 2D). This implies that, in studies of electrophysiological connectivity, the commonly measured electrophysiological frequencies should not be treated in isolation, but as part of a larger interactive multi-layer network where within and between band couplings are taken into account.

\section{Decomposing within frequency band networks}

Eigenvalue decomposition was undertaken to examine similarities and differences in the spatial signatures of within frequency band networks. Spatial patterns corresponding to the first two eigenmode products of each layer are shown in Fig. 3, panels A and B show the first and second eigenmode products, respectively. The first eigenmode product shows frequency specific structure within each of the 5 layers, whereas the second eigenmode product shows greater similarity across layers (see also Figs. S1 and S2).

The result in Fig. 3 is further supported by Fig. 4, which shows not only a relationship between layers, but also how this relationship 
first eigenmode products

A

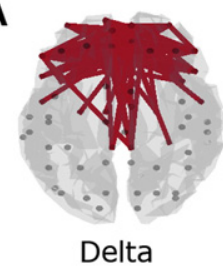

B

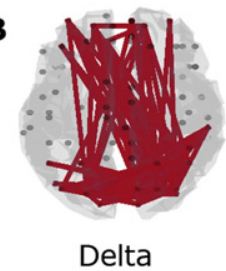

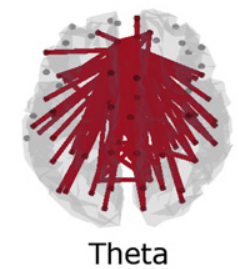

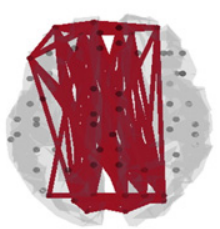

Theta

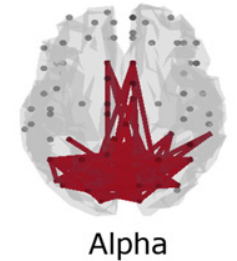

Alpha

second eigenmode products

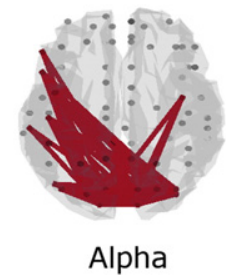

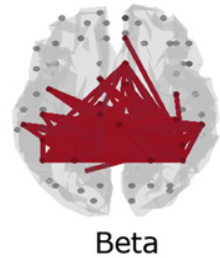

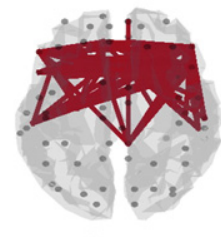

Gamma

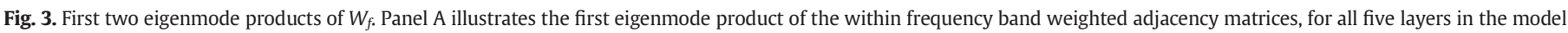

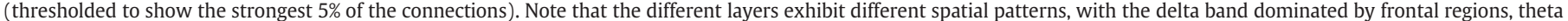

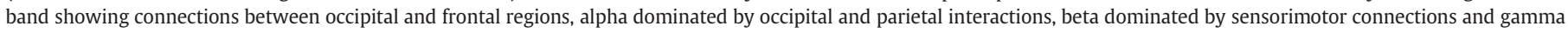

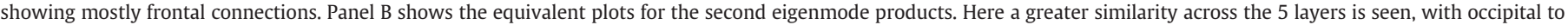

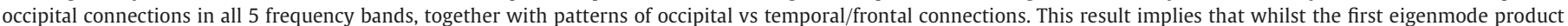
represents strong frequency dependent structure, the second eigenmode product shows a degree of spatial similarity between layers.

correlates with inter-layer coupling. Fig. 4A shows the average correlation across layers (i.e. the mean of $C\left(W_{l}, W_{f}\right)$ across all $l$ and $f$ ) plotted as a function of the eigenmode product that was removed. The value zero on the $\mathrm{x}$-axis corresponds to the condition where no eigenmode products were removed; the subsequent values show removal of the first, second, third and so on, eigenmode products from all layers. Removal of the first eigenmode product led to a sharp increase in global correlation, indicating that this eigenmode product is likely responsible for frequency specific information (Fig. 4A). In contrast, setting the second eigenmode product to zero led to a drop in global correlation between frequencies, indicating that this eigenmode product is likely to represent common structure seen across frequencies (Fig. 4B). Further supporting this picture, Fig. 4B shows correlations between layers for all possible layer pairs and is computed using the original weighted adjacency matrices $W_{f}$ (i.e. separate matrix elements show $C\left(W_{l}, W_{f}\right)$ for all $l$ and $f$ independently); Fig. 4C shows the same correlation values but computed in the absence of the first eigenmode product (i.e. separate elements show $C\left(W_{l}^{\prime}, W_{f}^{\prime}\right)$ ). Notice the increase in correlation values when the first eigenmode product is removed. Fig. $4 \mathrm{D}$ and $\mathrm{E}$ allows direct visualisation of this effect, with Fig. 4D showing the original weighted adjacency matrices for all layers and Fig. 4E showing the residual weighted adjacency matrices following removal of the first eigenmode product. There is more visual similarity between the residual weighted adjacency matrices than between the original weighted adjacency matrices. The high similarity between the residual weighted adjacency matrices raises the question of whether this similarity may be driven by the underlying structural network. Since the literature based structural network is an unweighted network, we thresholded the average residual matrix (mean across frequencies) based on exactly the same link density as the structural network. The fraction of common links between this thresholded average residual matrix and the literature based structural network is 0.47 .

Fig. 4F, $\mathrm{G}$ and $\mathrm{H}$ shed light on how the relationship between layers is affected by inter-layer coupling (the latter being estimated via $P$ ). Fig. $4 \mathrm{~F}$ shows inter-layer coupling strength $P$ plotted against correlation between layers (using the original weighted adjacency matrices for individual subjects). The significant positive correlation between $P$ and $C\left(W_{l}, W_{f}\right)$ (Pearson $R=0.7$, comparison to null-distribution $p=$ 0.008 ) reveals that those subjects who exhibit the strongest interlayer coupling also tend to exhibit the highest between layer similarity.
Interestingly, this significant relationship becomes even stronger following removal of the first eigenmode product for all layer weighted adjacency matrices (Fig. 4G; $R=0.88$, comparison to null-distribution $p=0.001$ ). Apart from the strength of the correlation, the increase in the correlation from $R=0.7$ to $R=0.88$ is also significant when compared to an increase in correlation values obtained for the same relationships from the null distribution $(p=0.008)$. In contrast, the correlation is much weaker when taking into account the first eigenmode product only (Fig. $4 \mathrm{H} ; R=0.45$, comparison to null-distribution $p=0.04$ ). Collectively Figs. 3 and 4 build further on the multi-layer network picture of MEG connectivity. The results suggest that the layers themselves are constructed from a superposition of layer specific spatial signatures, alongside common spatial signatures. Furthermore, these independent spatial patterns can be accessed via eigenvalue decomposition of the weighted adjacency matrices. In addition, for any one individual, the strength of the spatial similarity of networks described by each layer is (positively) related to the strength of inter-layer (cross-frequency) coupling.

\section{Global multi-layer network behaviour}

From the outset we formed a hypothesis that the operating point of MEG multi-layer networks exists close to a transition between independent and highly correlated layers. Here, we test this by comparing empirical data to simulated multi-layer networks (Network simulations based on a cortico-thalamic mean-field model section). Fig. 5A (blue trace) shows the simulated inter-layer coupling, $P$ as a function of structural coupling strength $\epsilon$ in the simulation. There are two regimes, characterised by a plateau of low values of $P$ for weak structural coupling and a plateau of high values of $P$ for strong structural coupling. Between these plateaus we observe a relatively steep transition between the two regimes. In addition, the correlation between the simulated and the empirical block-adjacency matrix is strongest for coupling strengths at the transition (green curve in Fig. 5A). For comparison, the red shaded curve shows the correlation (mean and standard deviation) between simulated and surrogate block-adjacency matrices. Similarly, the distribution of $P$ in the empirical MEG data is also optimally captured by the model in this transition region (green curve in Fig. 5B). Again MEG data outperforms the surrogate data (red shaded curve representing the mean and the standard deviation). The mean 
A

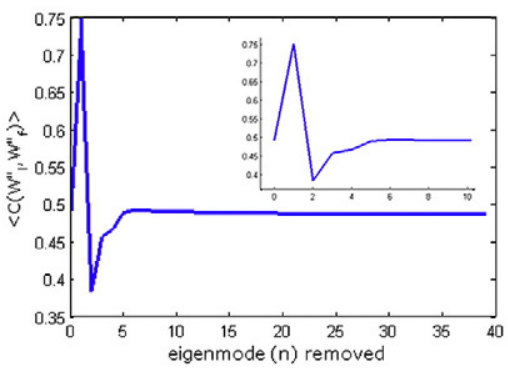

D

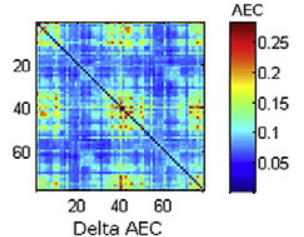

$\mathbf{E}$

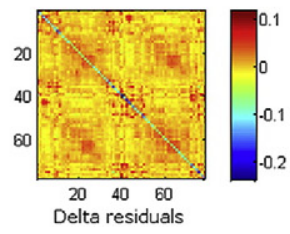

B

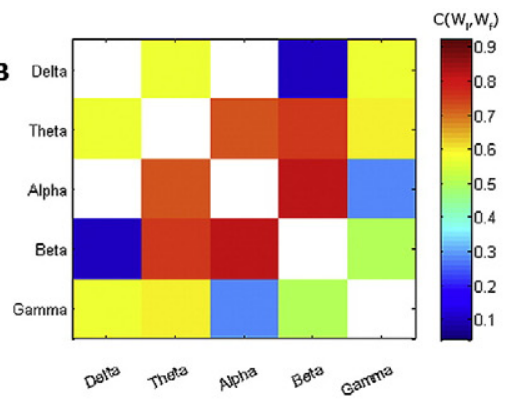

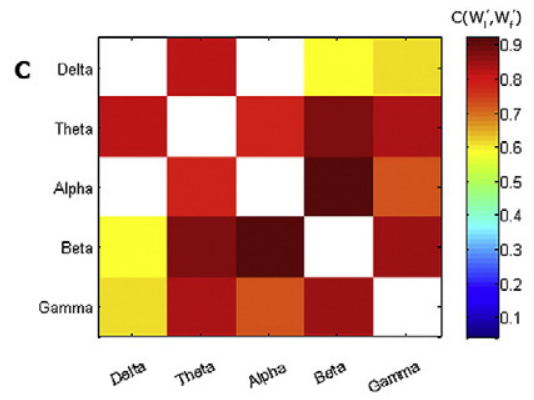

C
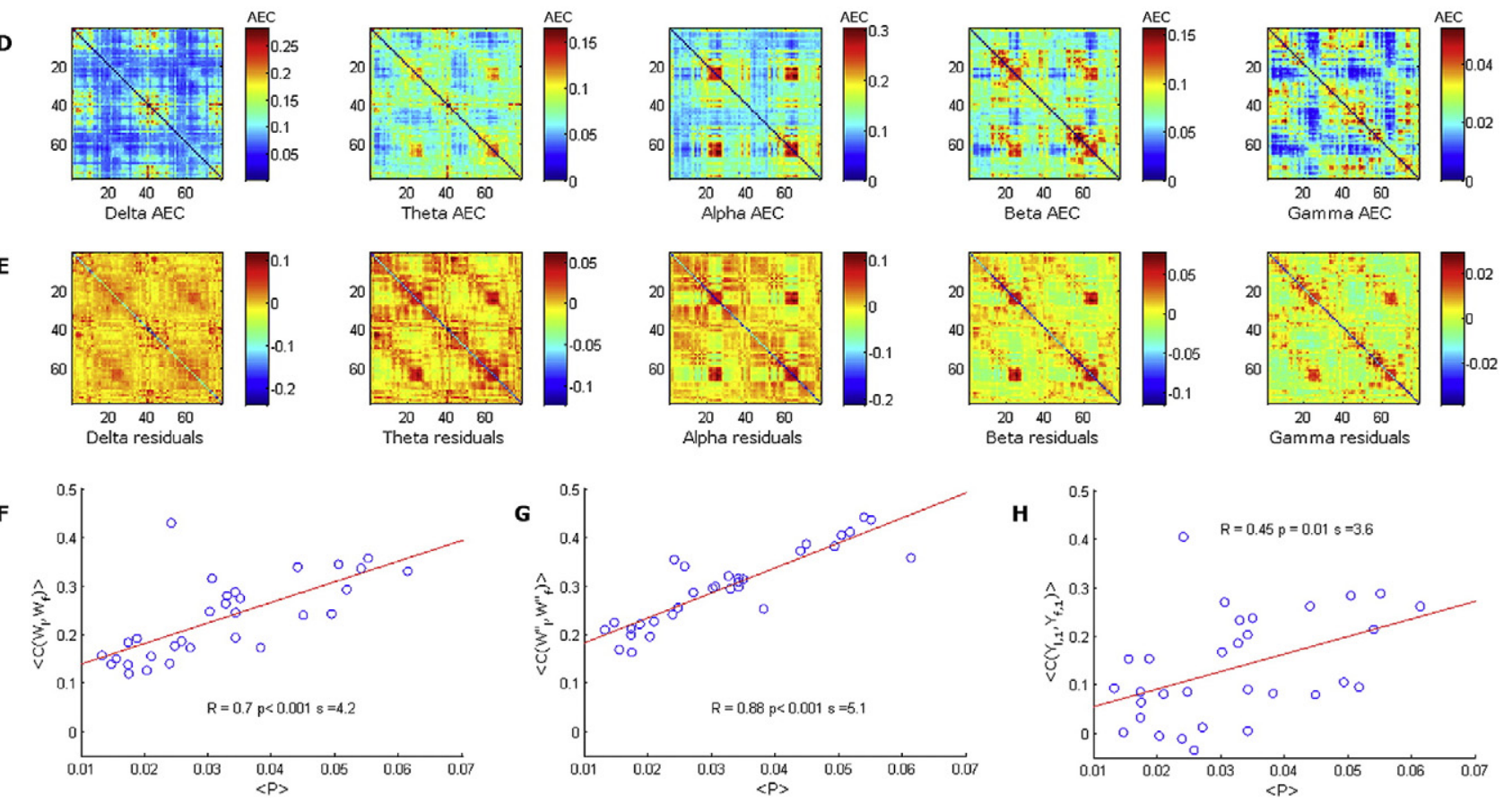

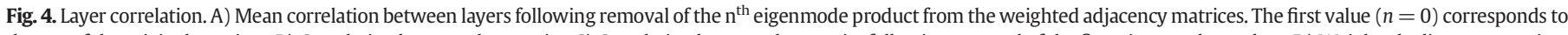

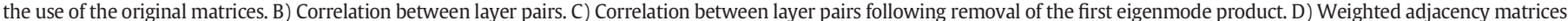

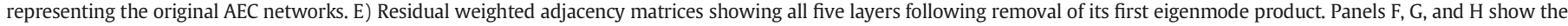

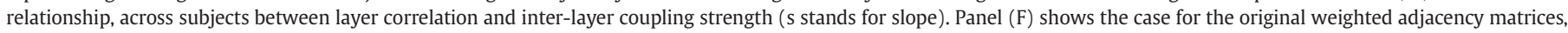

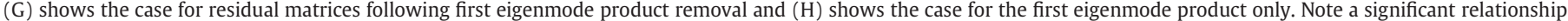

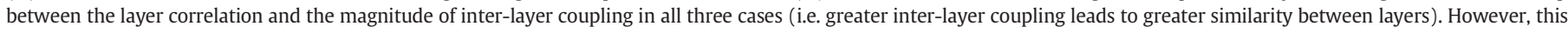
relationship is stronger if the first eigenvalue is removed.

(absolute) value of $P$ in the experimental data is significantly higher than for surrogate data (Mann-Whitney $U=-9.75, p<0.001$ ) (Fig. 5C), and the mean and range of the experimental $P$ match the values that were observed in the regime around the transition in the modelled data (Fig. 5A, C), which is not the case for the surrogate data. Lastly, when looking at the distribution of the eigenvalues of the block-Laplacian matrix (blue shaded region in Fig. 5D), the optimal fit is also found at the transition (green curve in Fig. 5D). The implication of this operational point for MEG multi-layer networks around a transition are twofold: 1 ) it allows for flexibility of $P$, i.e. dynamical switching between low and high values of $P$ (see SI and Fig. S3); and 2) it allows for integration and segregation between layers since the weak structural coupling regime corresponds to segregation of layers and the strong structural coupling regime to integration between layers (see Fig. 5E). Fig. 5E shows the conditional probability that links in layer $f$ are there given the presence of links in layer $m$. Similar to the eigenvalues and $P$ there are two plateaus separated by a transition when global coupling strength is tuned. The first plateau corresponds to low conditional probability values, indicating that segregation of layers, whereas the second plateau corresponds to high conditional probability and integration between layers. The transition thus corresponds to a mixed regime of segregation and integration between layers.
Fig. 5F, G and $\mathrm{H}$ shows example block-adjacency matrices derived from the model. Fig. 5F shows the case for low $P$, Fig. 5H shows the case for high $P$, and Fig. 5G shows the case for the transition. Note the visual similarity between Fig. 5G and Fig. 2B, which shows the experimental block-adjacency matrix. However, also note that the diagonal matrices in Fig. 2B contain more frequency specific structure than the simulated diagonal matrices. Fig. $5 \mathrm{H}$ shows a configuration, where all the nodes are almost fully synchronised in the within band layers.

\section{Discussion}

We have described a multi-layer framework that allows a complete spectral picture of network interactions. Using this framework, we have made some interesting observations: 1) the magnitude of within-layer (within frequency) and inter-layer (cross-frequency) coupling is positively correlated. Furthermore, the spatial similarity between networks in separate frequency bands depends on the strength of cross-frequency coupling; 2) separate layers are characterised by a common spatial mode, which is overlaid by a frequency specific mode with distinct spatial topographies. Cross-frequency coupling is more likely to play a role in mediating spatial similarity (i.e. the common mode) seen across-frequency bands; and 3) by combining empirical neuroimaging 

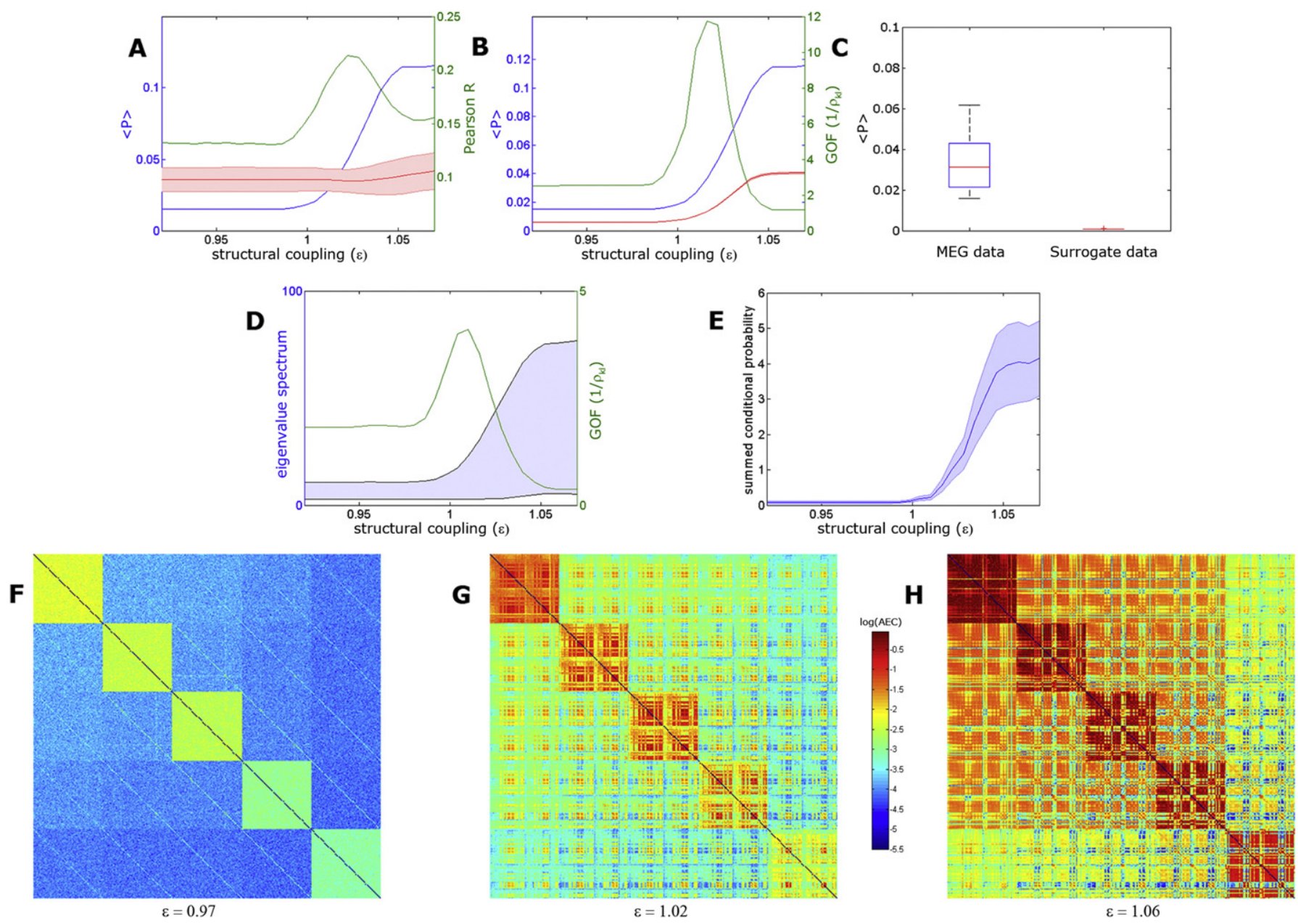

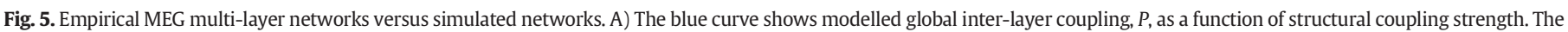

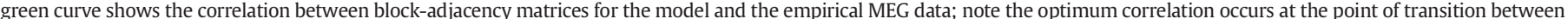

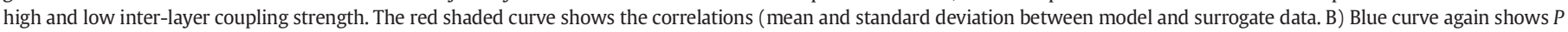

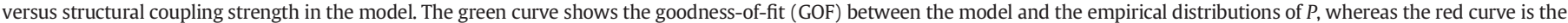

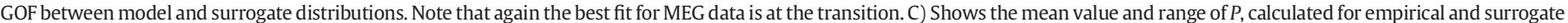

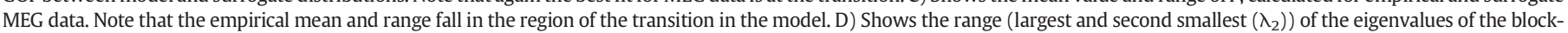

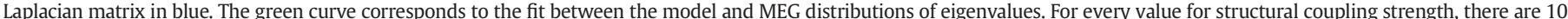

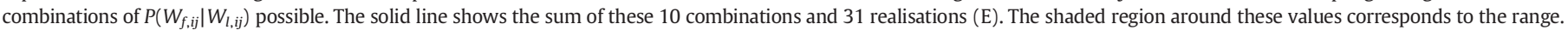

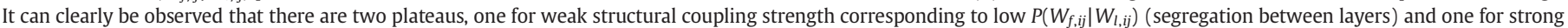

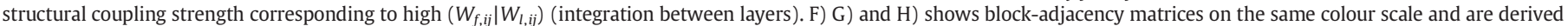
from the model for different coupling strengths.

data with a whole brain simulation of neuronal network activity, we observe a transition between two regimes of multi-layer network similarly as previously described in the network literature (Sahneh et al., 2015; Shakeri et al., 2015; Van Mieghem, 2016). The results suggest that the healthy human brain operates at the transition point between these regimes, allowing it to switch between two configurations, one in which different layers are independent and a second in which there is strong dependence between layers.

Previous studies have typically analysed either within frequency functional networks, or cross-frequency interactions in isolation. In our recent work we introduced the multi-layer network framework to integrate information of networks from different frequency bands in a single framework (Brookes et al., 2016). Here, we extend this previous work by analysing the relationship between inter- and intra-layer connections in more detail. Our empirical data clearly show that within and between frequency interactions are significantly correlated. This finding has potentially important implications, not only for our model of network interactions, but also for future studies of electrophysiological connectivity. Firstly, we found that the strength of inter-layer coupling significantly correlated with the averaged magnitude of within layer interactions. This non-trivial finding highlights that within and between frequency interactions should not be treated separately, but rather integrated into a broader picture of brain function. Secondly, the strength of cross-frequency (inter-layer) coupling strongly influenced the spatial network similarity across different layers (Fig. 4); i.e. if inter-layer coupling is high, the spatial connectivity patterns within the different layers become increasingly similar. Interestingly, this relationship was most prominent when considering residual networks following removal of the (frequency band specific) first eigenmode product from the original weighted adjacency matrices. Therefore, inter-layer coupling might play a more important role in mediating spatial similarity seen acrossfrequency bands, rather than allowing for communication between frequency specific independent spatial patterns. Note that the residual matrices show roughly $50 \%$ overlap with connections of a literature based structural network, indicating that the structural network might be an additional important factor that shapes this common mode. However, future studies with DTI and MEG data within the same subjects might provide more insight into the effect of the structural network on cross-frequency networks. A practical implication of the current findings is that, even if future studies on neurological diseases opt not to 
implement a multi-layer framework, they have to consider whether it is justified to treat networks obtained from different frequencies independently in their statistical tests (Demuru et al., 2014; Dimitriadis et al., 2015; Engels et al., 2015; Olde Dubbelink et al., 2013; Pang et al., 2015; Van Dellen et al., 2013).

The first eigenmode products for the different frequency bands showed frequency specific patterns of connectivity (Fig. 3A), and showed familiarity with patterns usually observed in frequency specific resting state MEG weighed adjacency matrices. For example, the first eigenmode product for the beta band was characterised by sensorimotor connections, whilst for the delta band the first eigenmode product was dominated by frontal connections. Importantly, the spatial patterns in the first eigenmode products for the theta and alpha band show striking similarity with recent findings on directed connectivity patterns in the upper alpha and theta band (Hillebrand et al., 2016). For the theta band, there is a divergent pattern of connections potentially originating from frontal regions. Although, we assessed connectivity and not information flow, a directed connectivity study has indeed shown that there is dominant flow of information from frontal regions to posterior and temporal regions in the theta band (Hillebrand et al., 2016). Likewise, the observed pattern in the alpha band (connections within posterior regions and between posterior and anterior regions) strongly resembles the posterior-to-anterior pattern in the upper alpha band in the directed connectivity study (Hillebrand et al., 2016), with the posterior regions as drivers of the information flow. Given the similarity between these directed connectivity findings and the current findings, it may be interesting for future studies to include directionality within a multi-layer network framework.

Regarding the global properties of our MEG derived multi-layer network, three findings in the current study point towards the idea that the operating point can be found near the transition between two regimes. Firstly, layers in the MEG multi-layer network were neither independent from each other nor completely identical. They were rather characterised by a common mode of connection patterns seen across all layers, superimposed by layer (frequency) specific patterns of connections. It is important to stress that such a common mode could not be found in surrogate data. Secondly, simulations showed that empirical MEG multi-layer networks could be found at the transition between two plateaus of inter-layer coupling strength when there was weak structural coupling. Thirdly, this also applied to the eigenvalues of the block-Laplacian matrix of MEG multi-layer networks, which were maximally explained by the model in the transition between the two regimes. Importantly, the modelling findings are in line with single frequency MEG network observations, where weakly coupled oscillators also outperformed other regimes of coupling in explaining empirical MEG data (Cabral et al., 2014; Nakagawa et al., 2014; Tewarie et al., 2014). The implication of this finding is that this operating point, at the boundary of integration and segregation of layers, likely allows the brain to switch rapidly between dependent and independent networks (see Fig. S3), thus giving rise to a 'dynamical flexibility' that would otherwise not be possible. We speculate that it is this dynamic capability that facilitates the flexibility necessary for the brain to generate, simultaneously and rapidly, a hierarchy of transient and temporally sustained networks. Here we examined this relationship in resting state eyesopen data only, and using networks derived across all time (meaning an entire resting state run). However, recent work now aims to understand the dynamic functional human connectome (Allen et al., 2014; Baker et al., 2014; Hutchison et al., 2013; O'Neill et al., 2015; O'Neill et al., 2016). Our current results suggest that using a multi-layer approach is such studies would bring new insights. We speculate that, at different points in time (or perhaps during different task phases) one might see differing multi-layer network behaviour with, for example, inter-layer coupling moving closer to either the integrative or independent network regimes depending on current (cognitive) processing demand. This alternation of periods with high and low inter-layer coupling may be accompanied by alternation of network integration and segregation within layers. For instance, previous studies on nonstationary MEG networks have shown that temporal networks are characterised by alternated periods of segregation and integration (de Pasquale et al., 2015), for which it is believed that functional hubs play a crucial role. Future dynamic multi-layer studies may address this and analyse if multi-layer functional hubs coordinate dynamic within and between band integration. A multi-layer network approach might also benefit from a more spatially selective hypothesis (e.g. a physiologically informed inter-layer coupling matrix - see also below). The current multi-layer network framework could also have clinical implications. Future studies on neurological diseases could investigate if structural damage might cause a shift away from the operating point, leading to weaker cross-frequency interactions, and therefore less integration between frequency bands and potentially poorer cognition.

\section{Methodological considerations}

There are some components of our method that warrant discussion. Firstly, the cortical AAL parcellation that was chosen has been used successfully in multiple previous MEG investigations (Brookes et al., 2016; Tewarie et al., 2016). However, the multi-layer approach could be used with any cortical parcellation. It is noteworthy that the separate AAL regions vary markedly in size, meaning that our use of a single point spread function for weighting voxels across each region may mean that some regions are better represented than others. This potentially represents a limitation and future use of brain parcellations based directly on the MEG data may therefore prove instructive. Secondly, for source localisation, we chose to employ a beamformer technique. Beamforming is a popular method of inverse solution (Hillebrand et al., 2005) and has previously been shown to be particularly useful in the characterisation of neuronal oscillations. Furthermore, beamforming has been used successfully in the estimation of functional connectivity (Brookes et al., 2011; Hillebrand et al., 2012). The reason for the success of this algorithm in such studies has been addressed at length in previous papers; for the interested reader we refer to (Hillebrand and Barnes, 2005). However, we point out that other inverse solutions (e.g. (de Pasquale et al., 2012)) could be easily substituted for beamforming in the present processing pipeline. Thirdly, here we chose an envelope correlation procedure as our estimator of functional connectivity between regions. Computation of envelope correlations comes with negative outcome values. In order to avoid cancellations in the calculation of averages, we considered the absolute values of the correlation values. This will artificially increase the average in all weighted adjacency matrices. However, in the current work, we were mainly interested in correlations between the patterns in the adjacency matrices for which the average connectivity is irrelevant. Computing envelope correlations have been successful in elucidating electrophysiological networks of functional connectivity (Colclough et al., 2016). However, other methods are available (Pereda et al., 2005); these should not be considered competitor techniques, but rather they probe a different type of functional connectivity (Siegel et al., 2012). The most common alternative to envelope methods for within frequency band connectivity is to probe the existence of phase synchronization between regions. Phase based metrics typically exhibit slightly lower signal to noise ratio compared to envelope methods (Colclough et al., 2016), however it is possible that such approaches might yield extra information on within frequency component of a multi-layer network. The choice of connectivity metric is also important in the case of cross-frequency interactions. Here, we chose to employ envelope correlation to quantify interactions between frequency bands for three reasons: i) it is straightforward to compute; 2 ) it is directly comparable to within frequency band connectivity; and iii) previous work suggests cross frequency envelope correlations to be of significant neurophysiological interest (Jensen and Colgin, 2007). One might argue that envelope correlation may not be a good measure to capture crossfrequency coupling, since envelopes computed for different frequency 
bands could show overlap in spectral content. However, envelopes are shown to contain specific information about the underlying carrier oscillations (Hipp et al., 2012), which have dissimilar spectral content. We point out that cross-frequency coupling can also be quantified via phase-amplitude interactions (i.e. where the phase of a low frequency oscillation links to the amplitude of a high frequency oscillation) and phase-phase based techniques (e.g. n oscillations of one signal correspond to $\mathrm{m}$ oscillations of a second signal). The multi-layer network framework could therefore benefit significantly from the use of these metrics. Lastly, the Euclidean distances between two regions in the simulations were not based on the anatomical distance along white matter tracts, and therefore delays between, in particular, homologous interhemispheric connections were underestimated in the simulations. However, realistic delays are more important when studying temporal patterns of connectivity (Deco et al., 2013a), whereas in the current study we were interested in stationary spatial patterns of functional networks.

Finally, from a more theoretical point of view, it is possible that the multi-layer model itself could be expanded. For example, this framework allows for the integration of multiple metrics (phase/phase, phase/amplitude and amplitude/amplitude), multiple modalities e.g. (MEG/EEG/fMRI) and structural networks. For example, the multilayer network could be modified into a system with the structural network as a layer at the bottom superimposed by layers for low and high frequency band networks. Additionally, in the present analysis, we have explored the case of 'all-to-all' inter-layer coupling (i.e. B is a matrix of ones, allowing any node in layer 1 to influence any node in layer $\mathrm{m}$ ). From a neuroscientific viewpoint, this seems reasonable: local neuronal circuits can generate several frequencies simultaneously (Womelsdorf et al., 2014) and several studies have shown long-range cross-frequency coupling (Palva et al., 2010; Sauseng et al., 2008). For this reason, we argue that an all-to-all coupling is probably a better approximation of the underlying neurophysiology than, for example, oneto-one coupling (e.g. B is the identity matrix). In future work, it may prove instructive for specific subnetworks to use neuroscientifically informed prior limitations on $B$ to select specific inter-layer interactions.

\section{Conclusion}

In this paper we proposed a multi-layer network framework that reconstructs a complete pan-spectral picture of neural network interactions. Using this model, we have demonstrated a strong dependency between layers and inter-layer coupling, indicating that networks obtained in different frequency bands do not act as independent entities, but that similarity seen across different frequency bands is related to inter-layer coupling. Moreover, we have demonstrated using a cortico-thalamic model that there are two regimes of multi-layer network behaviour; one in which different layers are highly dependent and a second where layers are independent. The combination of empirical neuroimaging data with whole brain simulations of neuronal network activity suggests that the healthy human brain operates at the transition point between these regimes, allowing integration and segregation between layers. Overall, our observations show that a complete picture of global brain network connectivity requires integration of oscillations across the full frequency spectrum, as well as crossfrequency coupling.

Supplementary data to this article can be found online at http://dx. doi.org/10.1016/j.neuroimage.2016.07.057.

\section{Acknowledgements}

This work was funded by a Medical Research Council New Investigator Research Grant (MR/M006301/1) awarded to MJB. We also acknowledge Medical Research Council Partnership Grant (MR/K005464/1). Data used were collected as part of the University of Nottingham Multimodal Imaging Study in Psychosis, funded by the
Medical Research Council (MR/J01186X/1). We therefore express our thanks to all those involved in data collection, particularly Emma Hall, Sian Robson and Jyothika Kumar.

\section{References}

Allen, E.A., Damaraju, E., Plis, S.M., Erhardt, E.B., Eichele, T., Calhoun, V.D., 2014. Tracking whole-brain connectivity dynamics in the resting state. Cereb. Cortex 24, 663-676 (bhs352).

Baker, A.P., Brookes, M.J., Rezek, I.A., Smith, S.M., Behrens, T., Smith, P.J.P., Woolrich, M., 2014. Fast transient networks in spontaneous human brain activity. Elife 3, e01867.

Barnes, G.R., Hillebrand, A., Fawcett, I.P., Singh, K.D., 2004. Realistic spatial sampling for MEG beamformer images. Hum. Brain Mapp. 23, 120-127.

Battiston, F., Nicosia, V., Latora, V., 2014. Structural measures for multiplex networks. Phys. Rev. E 89, 032804.

Benjamini, Y., Hochberg, Y., 1995. Controlling the false discovery rate: a practical and powerful approach to multiple testing. J. R. Stat. Soc. Ser. B Methodol. 289-300.

Boccaletti, S., Bianconi, G., Criado, R., Del Genio, C.I., Gómez-Gardeñes, J., Romance, M., Sendiña-Nadal, I., Wang, Z., Zanin, M., 2014. The structure and dynamics of multilayer networks. Phys. Rep. 544, 1-122.

Brookes, M.J., Hale, J.R., Zumer, J.M., Stevenson, C.M., Francis, S.T., Barnes, G.R., Owen, J.P., Morris, P.G., Nagarajan, S.S., 2011. Measuring functional connectivity using MEG: methodology and comparison with fcMRI. Neurolmage 56, 1082-1104.

Brookes, M.J., Woolrich, M.W., Barnes, G.R., 2012. Measuring functional connectivity in MEG: a multivariate approach insensitive to linear source leakage. Neurolmage 63, 910-920.

Brookes, M.J., O'Neill, G.C., Hall, E.L., Woolrich, M.W., Baker, A., Corner, S.P., Robson, S.E., Morris, P.G., Barnes, G.R., 2014. Measuring temporal, spectral and spatial changes in electrophysiological brain network connectivity. NeuroImage 91, 282-299.

Brookes, M.J., Tewarie, P.K., Hunt, B.A., Robson, S.E., Gascoyne, L.E., Liddle, E.B., Liddle, P.F., Morris, P.G., 2016. A multi-layer network approach to MEG connectivity analysis. Neurolmage 132, 425-438.

Brookes, M.J., Vrba, J., Robinson, S.E., Stevenson, C.M., Peters, A.M., Barnes, G.R., Hillebrand, A., Morris, P.G., 2008. Optimising experimental design for MEG beamformer imaging. Neurolmage 39, 1788-1802.

Cabral, J., Luckhoo, H., Woolrich, M., Joensson, M., Mohseni, H., Baker, A., Kringelbach, M.L. Deco, G., 2014. Exploring mechanisms of spontaneous functional connectivity in MEG: how delayed network interactions lead to structured amplitude envelopes of band-pass filtered oscillations. NeuroImage 90, 423-435.

Colclough, G., Brookes, M.J., Tewarie, P., Smith, S.M., Woolrich, M., 2016. How Reliable Are MEG Resting-state Connectivity Metrics? submitted

Coombes, S., 2010. Large-scale neural dynamics: simple and complex. NeuroImage 52, 731-739.

da Silva, F.L., 2013. EEG and MEG: relevance to neuroscience. Neuron 80, 1112-1128.

De Domenico, M., Solé-Ribalta, A., Cozzo, E., Kivelä, M., Moreno, Y., Porter, M.A., Gómez, S., Arenas, A., 2013. Mathematical formulation of multilayer networks. Phys. Rev. $\times 3$, 041022.

de Pasquale, F., Della Penna, S., Snyder, A.Z., Marzetti, L., Pizzella, V., Romani, G.L., Corbetta, M., 2012. A cortical core for dynamic integration of functional networks in the resting human brain. Neuron 74, 753-764.

de Pasquale, F., Della Penna, S., Sporns, O., Romani, G., Corbetta, M., 2015. A dynamic core network and global efficiency in the resting human brain. Cereb. Cortex bhv185.

Deco, G., Jirsa, V.K., McIntosh, A.R., 2013a. Resting brains never rest: computational insights into potential cognitive architectures. Trends Neurosci. 36, 268-274.

Deco, G., Ponce-Alvarez, A., Mantini, D., Romani, G.L., Hagmann, P., Corbetta, M., 2013b. Resting-state functional connectivity emerges from structurally and dynamically shaped slow linear fluctuations. J. Neurosci. 33, 11239-11252.

Deco, G., Tononi, G., Boly, M., Kringelbach, M.L., 2015. Rethinking segregation and integration: contributions of whole-brain modelling. Nat. Rev. Neurosci. 16, 430-439.

Demuru, M., van Duinkerken, E., Fraschini, M., Marrosu, F., Snoek, F.J., Barkhof, F., Klein, M., Diamant, M., Hillebrand, A., 2014. Changes in MEG resting-state networks are related to cognitive decline in type 1 diabetes mellitus patients. NeuroImage: Clin. 5 , 69-76.

Dimitriadis, S.I., Zouridakis, G., Rezaie, R., Babajani-Feremi, A., Papanicolaou, A.C., 2015. Functional connectivity changes detected with magnetoencephalography after mild traumatic brain injury. NeuroImage: Clin. 9, 519-531.

Dorogovtsev, S.N., Goltsev, A.V., Mendes, J.F., Samukhin, A.N., 2003. Spectra of complex networks. Phys. Rev. E 68, 046109.

Engel, A.K., Gerloff, C., Hilgetag, C.C., Nolte, G., 2013. Intrinsic coupling modes: multiscale interactions in ongoing brain activity. Neuron 80, 867-886.

Engels, M.M., Stam, C.J., van der Flier, W.M., Scheltens, P., de Waal, H., van Straaten, E.C., 2015. Declining functional connectivity and changing hub locations in Alzheimer's disease: an EEG study. BMC Neurol. 15, 145.

Freyer, F., Roberts, J.A., Becker, R., Robinson, P.A., Ritter, P., Breakspear, M., 2011. Biophysical mechanisms of multistability in resting-state cortical rhythms. J. Neurosci. 31, 6353-6361.

Gomez, S., Diaz-Guilera, A., Gomez-Gardeñes, J., Perez-Vicente, C.J., Moreno, Y., Arenas, A., 2013. Diffusion dynamics on multiplex networks. Phys. Rev. Lett. 110, 028701

Gong, G., He, Y., Concha, L., Lebel, C., Gross, D.W., Evans, A.C., Beaulieu, C., 2009. Mapping anatomical connectivity patterns of human cerebral cortex using in vivo diffusion tensor imaging tractography. Cereb. Cortex 19, 524-536.

Granell, C., Gómez, S., Arenas, A., 2013. Dynamical interplay between awareness and epidemic spreading in multiplex networks. Phys. Rev. Lett. 111, 128701.

Hall, E.L., Robson, S.E., Morris, P.G., Brookes, M.J., 2014. The relationship between MEG and fMRI. NeuroImage 102, 80-91. 
Hillebrand, A., Barnes, G.R., 2005. Beamformer analysis of MEG data. Int. Rev. Neurobiol. $68,149-171$.

Hillebrand, A., Singh, K.D., Holliday, I.E., Furlong, P.L., Barnes, G.R., 2005. A new approach to neuroimaging with magnetoencephalography. Hum. Brain Mapp. 25, 199-211.

Hillebrand, A., Barnes, G.R., Bosboom, J.L., Berendse, H.W., Stam, C.J., 2012. Frequencydependent functional connectivity within resting-state networks: an atlas-based MEG beamformer solution. Neurolmage 59, 3909-3921.

Hillebrand, A., Tewarie, P., van Dellen, E., Yu, M., Carbo, E.W.S., Douw, L., Gouw, A.A., van Straaten, E.C.W., Stam, C., 2016. Direction of information flow in large-scale restingstate networks is frequency dependent. Proc. Nat. Acad. Sci. 113, 3867-3872.

Hindriks, R., van Putten, M.J., 2012. Meanfield modeling of propofol-induced changes in spontaneous EEG rhythms. NeuroImage 60, 2323-2334.

Hindriks, R., van Putten, M.J., 2013. Thalamo-cortical mechanisms underlying changes in amplitude and frequency of human alpha oscillations. Neurolmage 70, 150-163.

Hipp, J.F., Hawellek, D.J., Corbetta, M., Siegel, M., Engel, A.K., 2012. Large-scale cortical correlation structure of spontaneous oscillatory activity. Nat. Neurosci. 15, 884-890.

Huang, M., Mosher, J., Leahy, R., 1999. A sensor-weighted overlapping-sphere head model and exhaustive head model comparison for MEG. Phys. Med. Biol. 44, 423.

Hutchison, R.M., Womelsdorf, T., Allen, E.A., Bandettini, P.A., Calhoun, V.D., Corbetta, M., Della Penna, S., Duyn, J.H., Glover, G.H., Gonzalez-Castillo, J., 2013. Dynamic functional connectivity: promise, issues, and interpretations. Neurolmage 80, 360-378.

Jensen, O., Colgin, L.L., 2007. Cross-frequency coupling between neuronal oscillations. Trends Cogn. Sci. 11, 267-269.

Jiang, H., Bahramisharif, A., van Gerven, M.A., Jensen, O., 2015. Measuring directionality between neuronal oscillations of different frequencies. NeuroImage 118, 359-367.

Liu, D., Wang, H., Van Mieghem, P., 2010. Spectral perturbation and reconstructability of complex networks. Phys. Rev. E 81, 016101.

Martín-Hernández, J., Wang, H., Van Mieghem, P., D'Agostino, G., 2014. Algebraic connectivity of interdependent networks. Phys. A: Stat. Mech. Appl. 404, 92-105.

Menichetti, G., Remondini, D., Panzarasa, P., Mondragón, R.J., Bianconi, G., 2014. Weighted Multiplex Networks.

Nakagawa, T.T., Woolrich, M., Luckhoo, H., Joensson, M., Mohseni, H., Kringelbach, M.L. Jirsa, V., Deco, G., 2014. How delays matter in an oscillatory whole-brain spikingneuron network model for MEG alpha-rhythms at rest. NeuroImage 87, 383-394.

Newman, M.E., 2006a. Finding community structure in networks using the eigenvectors of matrices. Phys. Rev. E 74, 036104.

Newman, M.E., 2006b. Modularity and community structure in networks. Proc. Natl. Acad. Sci. $103,8577-8582$.

Nicosia, V., Latora, V., 2014. Measuring and Modelling Correlations in Multiplex Networks arXiv preprint arXiv:1403.1546.

Nicosia, V., Bianconi, G., Latora, V., Barthelemy, M., 2013. Growing multiplex networks. Phys. Rev. Lett. 111, 058701.

O'Neill, G.C., Barratt, E.L., Hunt, B.A., Tewarie, P.K., Brookes, M.J., 2015. Measuring electrophysiological connectivity by power envelope correlation: a technical review on MEG methods. Phys. Med. Biol. 60, R271.

Olde Dubbelink, K.T., Stoffers, D., Deijen, J.B., Twisk, J.W., Stam, C.J., Hillebrand, A Berendse, H.W., 2013. Resting-state functional connectivity as a marker of disease progression in Parkinson's disease: a longitudinal MEG study. NeuroImage: Clin. 2, 612-619.

O'Neill, G.C., Bauer, M., Woolrich, M.W., Morris, P.G., Barnes, G.R., Brookes, M.J., 2015. Dynamic recruitment of resting state sub-networks. Neurolmage 115, 85-95.

O'Neill, G.C., Tewarie, P., Colclough, G., Gascoyne, L.E., Hunt, B., Morris, P.G., Woolrich, M., Brookes, M.J., 2016. Measurement of Dynamic Task Related Functional Networks Using MEG (in submission).

Palva, J.M., Monto, S., Kulashekhar, S., Palva, S., 2010. Neuronal synchrony reveals working memory networks and predicts individual memory capacity. Proc. Natl. Acad. Sci. 107, 7580-7585

Pang, E.W., Dunkley, B.T., Doesburg, S.M., da Costa, L., Taylor, M.J., 2015. Reduced brain connectivity and mental flexibility in mild traumatic brain injury. Ann. Clin. Trans. Neurol. 3, 124-131.

Pereda, E., Quiroga, R.Q., Bhattacharya, J., 2005. Nonlinear multivariate analysis of neurophysiological signals. Prog. Neurobiol. 77, 1-37.

Prichard, D., Theiler, J., 1994. Generating surrogate data for time series with several simultaneously measured variables. Phys. Rev. Lett. 73, 951.

Radicchi, F., Arenas, A., 2013. Abrupt transition in the structural formation of interconnected networks. Nat. Phys. 9, 717-720.

Robinson, S., Vrba, J., 1999. Functional neuroimaging by synthetic aperture magnetometry (SAM). Recent adv. Biomagn. 302-305.
Robinson, P., Rennie, C., Wright, J., Bahramali, H., Gordon, E., Rowe, D., 2001. Prediction of electroencephalographic spectra from neurophysiology. Phys. Rev. E 63, 021903.

Robinson, P., Rennie, C., Rowe, D., 2002. Dynamics of large-scale brain activity in normal arousal states and epileptic seizures. Phys. Rev. E 65, 041924.

Robinson, P., Rennie, C., Rowe, D., O'Connor, S., 2004. Estimation of multiscale neurophysiologic parameters by electroencephalographic means. Hum. Brain Mapp. 23, 53-72.

Sahneh, F.D., Scoglio, C., Van Mieghem, P., 2015. Exact coupling threshold for structura transition reveals diversified behaviors in interconnected networks. Phys. Rev. E 92, 040801.

Sánchez-García, R.J., Cozzo, E., Moreno, Y., 2014. Dimensionality reduction and spectral properties of multilayer networks. Phys. Rev. E 89, 052815.

Sarvas, J., 1987. Basic mathematical and electromagnetic concepts of the biomagnetic inverse problem. Phys. Med. Biol. 32, 11.

Sauseng, P., Klimesch, W., Gruber, W.R., Birbaumer, N., 2008. Cross-frequency phase synchronization: a brain mechanism of memory matching and attention. NeuroImage 40, 308-317.

Schölvinck, M.L., Leopold, D.A., Brookes, M.J., Khader, P.H., 2013. The contribution of electrophysiology to functional connectivity mapping. Neurolmage 80, 297-306.

Shakeri, H., Albin, N., Sahneh, F.D., Poggi-Corradini, P., Scoglio, C., 2015. Maximizing Algebraic Connectivity in Interconnected Networks arXiv preprint arXiv:1510.06785.

Siegel, M., Donner, T.H., Engel, A.K., 2012. Spectral fingerprints of large-scale neuronal interactions. Nat. Rev. Neurosci. 13, 121-134.

Sole-Ribalta, A., De Domenico, M., Kouvaris, N.E., Diaz-Guilera, A., Gomez, S., Arenas, A., 2013. Spectral properties of the Laplacian of multiplex networks. Phys. Rev. E 88 032807.

Stam, C.J., 2014. Modern network science of neurological disorders. Nat. Rev. Neurosci. 15 683-695.

Tewarie, P., Hillebrand, A., van Dellen, E., Schoonheim, M., Barkhof, F., Polman, C., Beaulieu, C., Gong, G., van Dijk, B., Stam, C., 2014. Structural degree predicts functional network connectivity: a multimodal resting-state fMRI and MEG study. Neurolmage 97, 296-307.

Tewarie, P., Schoonheim, M.M., Schouten, D.I., Polman, C.H., Balk, L.J., Uitdehaag, B.M. Geurts, J.J., Hillebrand, A., Barkhof, F., Stam, C.J., 2015. Functional brain networks: linking thalamic atrophy to clinical disability in multiple sclerosis, a multimodal fMRI and MEG study. Hum. Brain Mapp. 36, 603-618.

Tewarie, P., Bright, M., Hillebrand, A., Robson, S., Gascoyne, L., Morris, P., Meier, J., Van Mieghem, P., Brookes, M., 2016. Predicting haemodynamic networks using electrophysiology: the role of non-linear and cross-frequency interactions. NeuroImage $130,273-292$

Tzourio-Mazoyer, N., Landeau, B., Papathanassiou, D., Crivello, F., Etard, O., Delcroix, N. Mazoyer, B., Joliot, M., 2002. Automated anatomical labeling of activations in SPM using a macroscopic anatomical parcellation of the MNI MRI single-subject brain. Neurolmage 15, 273-289.

Van Dellen, E., de Witt Hamer, P., Douw, L., Klein, M., Heimans, J., Stam, C., Reijneveld, J. Hillebrand, A., 2013. Connectivity in MEG resting-state networks increases after resective surgery for low-grade glioma and correlates with improved cognitive performance. Neuroimage: Clin. 2, 1-7.

Van Mieghem, P., 2010. Graph Spectra for Complex Networks. Cambridge University Press.

Van Mieghem, P., 2014. Graph eigenvectors, fundamental weights and centrality metrics for nodes in networks. arXiv preprint arXiv 1401-4580.

Van Mieghem, P., 2016. Interconnectivity structure of a general interdependent network Phys. Rev. E 93, 042305.

Wang, X., Van Mieghem, P., 2015. Orthogonal Eigenvector Matrix of the Laplacian Fourth International IEEE Workshop on Complex Networks and their Applications, Bangkok, Thailand.

Wang, H., Li, Q., D'Agostino, G., Havlin, S., Stanley, H.E., Van Mieghem, P., 2013. Effect of the interconnected network structure on the epidemic threshold. Phys. Rev. E 88, 022801.

Watrous, A.J., Deuker, L., Fell, J., Axmacher, N., 2015. Phase-amplitude coupling supports phase coding in human ECoG. eLife 4, e07886.

Womelsdorf, T., Valiante, T.A., Sahin, N.T., Miller, K.J., Tiesinga, P., 2014. Dynamic circuit motifs underlying rhythmic gain control, gating and integration. Nat. Neurosci. 17 1031-1039.

Zalesky, A., Fornito, A., Bullmore, E., 2012. On the use of correlation as a measure of network connectivity. Neurolmage 60, 2096-2106. 